\title{
The invariant manifold structure of the spatial Hill's problem
}

\author{
G. GÓMEZ†*, M. MARCOTEł and J. M. MONDELO† \\ $\dagger$ IEEC \& Departament Matemàtica Aplicada i Anàlisi, Universitat de Barcelona, \\ Gran Via 585, 08007 Barcelona, Spain \\ fIEEC \& Departament Matemàtiques, Universitat Autònoma de Barcelona, \\ 08193 Bellaterra, Barcelona, Spain
}

(Received 16 April 2004; in final form 31 August 2004)

\begin{abstract}
The paper studies the invariant manifolds of the spatial Hill's problem associated to the two liberation points. A combination of analytical and numerical tools allow the normalization of the Hamiltonian and the computation of periodic and quasi-periodic (invariant tori) orbits. With these tools, it is possible to give a complete description of the center manifolds, association to the liberation points, for a large set of energy values.

A systematic exploration of the homoclinic and heteroclinic connections between the center manifolds of the liberation points is also given.
\end{abstract}

\section{Introduction}

This paper is devoted to the study of the centre manifolds of the two libration points of the 3D Hill's problem, as well as the invariant stable and unstable manifolds associated to them.

The motivation for the study comes from some previous work related to the invariant manifold structures of the collinear equilibrium points of the restricted three-body problem (RTBP) [1-4]. These manifolds, and the connections between them, not only provide the framework for understanding transport phenomena from a geometric point of view, but can also be used to get low energy transfers to and between the libration points, useful for some spacecraft mission designs such as Soho and Genesis (see [5-7]). The orbits giving those connections may provide the backbone for other useful spacecraft orbits in the future.

A complete and systematic study of the homoclinic and heteroclinic connections between the collinear libration point orbits in the RTBP has not been done. This is mainly due to the large number of computations that must be performed and the huge amount of data to be analysed. The explorations done up to now show a lot of different connections between the several kinds of orbits of the centre manifolds. To

*Corresponding author. Email: gerard@maia.ub.es

Dynamical Systems: An International Journal 
clarify the situation, it seems convenient to take as a starting point an approximation of the RTBP, such as Hill's problem, in which the symmetries allow a substantial reduction of the total amount of computations and data to be stored and analysed. This is the main purpose of the present paper, which is also an extension of the numerical study done in [8,9] for the planar Hill problem, and in which the geometrical behaviour of the centre-stable/unstable manifolds of the libration points was investigated.

\subsection{Equations of motion}

The spatial restricted problem of three bodies (RTBP) may be defined by

$$
\begin{aligned}
x^{\prime \prime}-2 y^{\prime} & =\Omega_{x}, \\
y^{\prime \prime}+2 x^{\prime} & =\Omega_{y}, \\
z^{\prime \prime} & =\Omega_{z},
\end{aligned}
$$

with

$$
\Omega(x, y, z)=\frac{1}{2}\left(x^{2}+y^{2}\right)+\frac{\mu}{\sqrt{(x+1-\mu)^{2}+y^{2}+z^{2}}}+\frac{1-\mu}{\sqrt{(x-\mu)^{2}+y^{2}+z^{2}}},
$$

where the origin of the rotating coordinate system $(x, y, z)$ is the centre of mass of the bodies with masses $m_{1}=1-\mu$ and $m_{2}=\mu$, which rest at the respective points $(\mu, 0,0)$ and $(\mu-1,0,0)$ (see [10]).

To study the motion in the vicinity of the small primary $m_{2}$, the origin of the coordinate system is transferred to the mass $\mu$, and the coordinates are scaled by a factor $\mu^{1 / 3}$, that is

$$
\begin{aligned}
& x=\mu^{1 / 3} \xi+\mu-1, \\
& y=\mu^{1 / 3} \eta, \\
& z=\mu^{1 / 3} \zeta .
\end{aligned}
$$

Substituting the inverse of this transformation into the equations of motion, and using the Taylor expansion of the last term of $\Omega$, one sees that (1) is again valid if (2) is replaced by

$$
\Omega=\frac{1}{2}\left(3 \xi^{2}-\zeta^{2}\right)+\frac{1}{\sqrt{\xi^{2}+\eta^{2}+\zeta^{2}}}+\mu^{1 / 3} \xi\left(\xi^{2}-\frac{3}{2}\left(\eta^{2}+\zeta^{2}\right)\right)+O\left(\mu^{2 / 3}\right) .
$$

Assume that the mass $\mu$ is very small, so we can neglect all the $O\left(\mu^{1 / 3}\right)$ terms of $\Omega(\xi, \zeta, \eta)$. In this situation, one gets the so-called Hill's limiting case of the restricted problem of three bodies (see [10-12])

$$
\begin{aligned}
\xi^{\prime \prime}-2 \eta^{\prime} & =3 \xi-\frac{\xi}{\left(\xi^{2}+\eta^{2}+\zeta^{2}\right)^{3 / 2}}, \\
\eta^{\prime \prime}+2 \xi^{\prime} & =-\frac{\eta}{\left(\xi^{2}+\eta^{2}+\zeta^{2}\right)^{3 / 2}}, \\
\zeta^{\prime \prime} & =-\zeta-\frac{\zeta}{\left(\xi^{2}+\eta^{2}+\zeta^{2}\right)^{3 / 2}} .
\end{aligned}
$$

In this limiting case, the large primary may be thought of as being situated at $x=+\infty$ of the $y=0$ axis, so system (4) has just one singularity at the origin, which is where the small primary has been located. 
Hill's problem equations can also be written in Hamiltonian form. The Hamiltonian function is

$$
\mathcal{H}=\frac{1}{2}\left(p_{x}^{2}+p_{y}^{2}+p_{z}^{2}\right)+q_{y} p_{x}-q_{x} p_{y}-\frac{1}{\sqrt{q_{x}^{2}+q_{y}^{2}+q_{z}^{2}}}-q_{x}^{2}+\frac{1}{2}\left(q_{y}^{2}+q_{z}^{2}\right),
$$

where $q_{x}=\xi, q_{y}=\eta, q_{z}=\zeta, p_{x}=\xi^{\prime}-\eta, p_{y}=\eta^{\prime}+\xi, p_{z}=\zeta^{\prime}$, is a canonical set of variables. From (5), it is clearly seen that $\mathcal{H}$ corresponds to a Kepler problem under the perturbation of the Coriolis force and the gravitational zero-order term in $\mu^{1 / 3}$.

As in the RTBP, the equations of motion have a first integral, the Jacobi integral, defined by

$$
C=3 \xi^{2}-\zeta^{2}+\frac{2}{\sqrt{\xi^{2}+\eta^{2}+\zeta^{2}}}-\left(\dot{\xi}^{2}+\dot{\eta}^{2}+\dot{\zeta}^{2}\right),
$$

whose value is related to that of the Hamiltonian by $C=-2 \mathcal{H}$.

\subsection{Symmetries and equilibrium points}

From the inspection of the terms occurring in the differential equations (4), it follows that if $(\xi(t), \eta(t), \zeta(t))$ is a particular solution, then

(i) $s_{1}(\xi(t), \eta(t), \zeta(t))=(\xi(-t),-\eta(-t), \zeta(-t))$,

(ii) $s_{2}(\xi(t), \eta(t), \zeta(t))=(-\xi(-t), \eta(-t), \zeta(-t))$,

(iii) $s_{3}(\xi(t), \eta(t), \zeta(t))=(\xi(t), \eta(t),-\zeta(t))$,

are also solutions. Two of these symmetries hold also for the RTBP.

Because of the limit location of one of the primaries, it is clear that the third collinear and both equilateral libration points of the RTBP disappear. Actually, it is readily found from (4) that this system has only two equilibrium points at

$$
L_{1}=(\xi, \eta, \zeta)=\left(3^{-1 / 3}, 0,0\right) \text { and } L_{2}=(\xi, \eta, \zeta)=\left(-3^{-1 / 3}, 0,0\right) \text {, }
$$

which obviously correspond to the first two of the three collinear libration points of the RTBP.

Due to the symmetry with respect to the $\xi=0$ plane, we can restrict the study to just one of the two points, for instance $L_{1}$. The analysis of the linear behaviour of the flow around this point shows that the eigenvalues are

$$
\begin{aligned}
& \lambda_{1,2}= \pm \sqrt{1+2 \sqrt{7}}=: \pm \lambda, \\
& \lambda_{3,4}= \pm \sqrt{1-2 \sqrt{7}}=: \pm \omega i, \\
& \lambda_{5,6}= \pm \sqrt{-4}=: \pm v i,
\end{aligned}
$$

so that the equilibrium point $L_{1}$ is saddle $\times$ centre $\times$ centre, like in the RTBP.

\subsection{Regularization of the singularity at the origin}

Using the Kustaanheimo-Stiefel (KS) regularization (see [13]) the singularity that the equations of motion have at the origin can be removed. Recall that this regularization, which is a generalization of the Levi-Civita transformation for the planar 
case, must necessarily be done increasing the number of degrees of freedom of the problem from three to four, due to topological obstructions. To introduce it, we will use the Hamiltonian formulation of the problem.

Let $\mathbf{u} \in \mathbb{R}^{4}$, the KS matrix, $L(\mathbf{u})$, is defined by

$$
L(\mathbf{u})=\left(\begin{array}{cccc}
u_{1} & -u_{2} & -u_{3} & u_{4} \\
u_{2} & u_{1} & -u_{4} & -u_{3} \\
u_{3} & u_{4} & u_{1} & u_{2} \\
u_{4} & -u_{3} & u_{2} & -u_{1}
\end{array}\right) .
$$

If $\mathbf{q}=\left(q_{x}, q_{y}, q_{z}\right)^{T} \in \mathbb{R}^{3}$, we define $\hat{\mathbf{q}}=\left(q_{x}, q_{y}, q_{z}, 0\right)^{T} \in \mathbb{R}^{4}$ and the KS transformation for the coordinates by

$$
\hat{\mathbf{q}}=L(\mathbf{u}) \mathbf{u} \text {. }
$$

The associated new momenta, w, are given by

$$
\hat{\mathbf{p}}=\frac{2}{r} L(\mathbf{u}) \mathbf{w},
$$

where $\hat{\mathbf{p}}=\left(p_{x}, p_{y}, p_{z}, 0\right)^{T}$ and $r=\sqrt{q_{x}^{2}+q_{y}^{2}+q_{z}^{2}}=u_{1}^{2}+u_{2}^{2}+u_{3}^{2}+u_{4}^{2}=\|\mathbf{u}\|^{2}$. In this way, the canonical transformation is given by

$$
\begin{array}{ll}
q_{x}=u_{1}^{2}-u_{2}^{2}-u_{3}^{2}+u_{4}^{2}, & p_{x}=\left(u_{1} w_{1}-u_{2} w_{2}-u_{3} w_{3}+u_{4} w_{4}\right) /(2 r), \\
q_{y}=2\left(u_{1} u_{2}-u_{3} u_{4}\right), & p_{y}=\left(u_{2} w_{1}+u_{1} w_{2}-u_{4} w_{3}-u_{3} w_{4}\right) /(2 r), \\
q_{z}=2\left(u_{1} u_{3}-u_{2} u_{4}\right), & p_{z}=\left(u_{3} w_{1}+u_{4} w_{2}+u_{1} w_{3}+u_{2} w_{4}\right) /(2 r) .
\end{array}
$$

A new independent variable, $s$, defined by

$$
\frac{\mathrm{d}}{\mathrm{d} t}=\frac{4}{r} \frac{\mathrm{d}}{\mathrm{d} s}
$$

must be used to complete the regularization. Following the homogeneous formalism described in [13] time is introduced as an additional coordinate whose associated momentum is $w_{0}=-h_{\mathcal{H}}\left(h_{\mathcal{H}}\right.$ being the value of the Hamiltonian $\left.\mathcal{H}\right)$. After performing the canonical transformation, we get for the new Hamiltonian

$$
H(\mathbf{u}, \mathbf{w})=\frac{r}{4}\left(\mathcal{H}(\mathbf{u}, \mathbf{w})+w_{0}\right),
$$

that can be written as

$$
\begin{aligned}
H= & \frac{w_{0}}{2} \frac{u_{1}^{2}+u_{2}^{2}+u_{3}^{2}+u_{4}^{2}}{2}+\frac{w_{1}^{2}+w_{2}^{2}+w_{3}^{2}+w_{4}^{2}}{2} \\
& +\frac{1}{2}\left(u_{1}^{2}+u_{2}^{2}\right)\left(u_{2} w_{1}-u_{1} w_{2}\right)+\frac{1}{2}\left(u_{1}^{2}-u_{2}^{2}\right)\left(u_{3} w_{3}+u_{4} w_{4}\right) \\
& +\frac{1}{2}\left(u_{3}^{2}+u_{4}^{2}\right)\left(u_{4} w_{3}-u_{3} w_{4}\right)+\frac{1}{2}\left(u_{3}^{2}-u_{4}^{2}\right)\left(u_{2} w_{2}+u_{1} w_{1}\right) \\
& +u_{1} u_{2}\left(u_{4} w_{4}-w_{3} u_{3}\right)+u_{3} u_{4}\left(u_{2} w_{2}-u_{1} w_{1}\right) \\
& -\frac{1}{4}\left[u_{1}^{6}+u_{2}^{6}+u_{3}^{6}+u_{4}^{6}-3 u_{1}^{4}\left(u_{2}^{2}+u_{3}^{2}-u_{4}^{2}\right)-3 u_{2}^{4}\left(u_{1}^{2}-u_{3}^{2}+u_{4}^{2}\right) .\right. \\
& -3 u_{3}^{4}\left(u_{1}^{2}-u_{2}^{2}+u_{4}^{2}\right)-3 u_{4}^{4}\left(-u_{1}^{2}+u_{2}^{2}+u_{3}^{2}\right) \\
& \left.-6\left(u_{1}^{2} u_{2}^{2} u_{3}^{2}+u_{1}^{2} u_{2}^{2} u_{4}^{2}+u_{1}^{2} u_{3}^{2} u_{4}^{2}+u_{2}^{2} u_{3}^{2}+u_{4}^{2}\right)\right]+\frac{1}{4} .
\end{aligned}
$$


To remove the dependence of $H$ on the parameter $w_{0}$ we introduce, as is done in [9] the canonical transformation

$$
u_{k}=\alpha Q_{k}, \quad w_{k}=\beta P_{k}, \quad k=1,2,3,4,
$$

and $H \equiv \gamma H$, where $\alpha=2\left(w_{0} / 2\right)^{1 / 4}, \beta=2\left(w_{0} / 2\right)^{3 / 4}$ and $\gamma=\left(w_{0} / 2\right)^{-3 / 2} / 4$. In this way, we have

$$
H\left(Q_{1}, Q_{2}, Q_{3}, Q_{4}, P_{1}, P_{2}, P_{3}, P_{4}\right)=H_{2}+H_{4}+H_{6},
$$

where

$$
\begin{aligned}
H_{2}= & \frac{1}{2}\left(Q_{1}^{2}+Q_{2}^{2}+Q_{3}^{2}+Q_{4}^{2}+P_{1}^{2}+P_{2}^{2}+P_{3}^{2}+P_{4}^{2}\right), \\
H_{4}= & 2\left(Q_{1}^{2}+Q_{2}^{2}\right)\left(Q_{2} P_{1}-Q_{1} P_{2}\right)+2\left(Q_{1}^{2}-Q_{2}^{2}\right)\left(Q_{3} P_{3}+Q_{4} P_{4}\right) \\
& +2\left(Q_{3}^{2}+Q_{4}^{2}\right)\left(Q_{4} P_{3}-Q_{3} P_{4}\right)+2\left(Q_{3}^{2}-Q_{4}^{2}\right)\left(Q_{1} P_{1}+Q_{2} P_{2}\right) \\
& +4 Q_{1} Q_{2}\left(Q_{4} P_{4}-Q_{3} P_{3}\right)+4 Q_{3} Q_{4}\left(Q_{2} P_{2}-Q_{1} P_{1}\right), \\
H_{6}= & -4\left[Q_{1}^{6}+Q_{2}^{6}+Q_{3}^{6}+Q_{4}^{6}-3 Q_{1}^{4}\left(Q_{2}^{2}+Q_{3}^{2}-Q_{4}^{2}\right)-3 Q_{2}^{4}\left(Q_{1}^{2}-Q_{3}^{2}+Q_{4}^{2}\right)\right. \\
& -3 Q_{3}^{4}\left(Q_{1}^{2}-Q_{2}^{2}+Q_{4}^{2}\right)-3 Q_{4}^{2}\left(-Q_{1}^{2}+Q_{2}^{2}+Q_{3}^{2}\right) \\
& \left.-6\left(Q_{1}^{2} Q_{2}^{2} Q_{3}^{2}+Q_{1}^{2} Q_{2}^{2} Q_{4}^{2}+Q_{1}^{2} Q_{3}^{2} Q_{4}^{2}+Q_{2}^{2} Q_{3}^{2} Q_{4}^{2}\right)\right] .
\end{aligned}
$$

If $Q_{3}=Q_{4}=0, P_{3}=P_{4}=0$ the Hamiltonian becomes equal to the one given in [9] for the regularized planar problem. As in this case, $H_{2}$ is the Kepler term and $H_{4}$ the Coriolis one. The remaining term, $H_{6}$, is the perturbation that breaks down the integrability of the system (see $[14,15]$ ).

The regularized Hamiltonian has now an equilibrium point (centre) at the origin and the two libration points $L_{1}$ and $L_{2}$ become two circles (in configuration space).

\section{Dynamics around the $L_{1}$ point}

Close to the $L_{1}$ libration point, the dynamics is that of an unstable equilibrium due to the saddle component of the linear approximation of the flow. However, due to the centre $\times$ centre part, and when all the energy levels are considered, there is a 4D centre manifold around $L_{1}$, which is also called the neutrally stable manifold. For a given energy level, it is a 3D set where the dynamics has a neutral behaviour. On these energy levels there are the periodic orbits and 2D tori.

For values of the Hamiltonian close to that of the equilibrium point, the analysis of the dynamics in the centre manifold can be done in a semi-analytical way. It mainly consists in performing a reduction of the Hamiltonian that decreases the number of degrees of freedom, removing the hyperbolic directions. The reduced Hamiltonian allows the numerical study of the Poincare map in the vicinity of the equilibrium point. This approach, which will be used in this section, is limited by the convergence of the expansions used (see $[16,17])$. To overcome this difficulty we can proceed to the direct numerical computation of the periodic orbits and $2 \mathrm{D}$ tori of the unreduced Hamiltonian. With this approach we can extend the analysis of the phase space to a wider range of energy values, that include several bifurcations. The methodology, and the corresponding results of this numerical approach, are given in section 6 . 


\subsection{Normal form around $\mathrm{L}_{1}$}

For the semi-analytical approach, the non-regularized Hamiltonian given in (5) will be used. As has been said, the regularization of the problem under consideration requires increasing the number of degrees of freedom from three to four. In this situation, and even after removing the saddle components, we do not have a 2-dimensional surface of section allowing a clear representation of the phase space. The regularized equations will be used for the globalization of the stable and unstable manifolds of the centre manifold, since they get very close to the origin.

The linear terms of the flow around the $L_{1}$ point are given by the second-order terms of (5)

$$
\mathcal{H}_{2}=\frac{1}{2}\left(p_{x}^{2}+p_{y}^{2}\right)+q_{y} p_{x}-q_{x} p_{y}-4 q_{x}^{2}+2 q_{y}^{2}+\frac{1}{2} p_{z}^{2}+2 q_{z}^{2} .
$$

The canonical transformation defined by the symplectic matrix

$$
\left(\begin{array}{cccccc}
2 \lambda / s & 0 & 0 & -2 \lambda / s & 2 \omega / t & 0 \\
\left(\lambda^{2}-9\right) / s & \left(-\omega^{2}-9\right) / t & 0 & \left(\lambda^{2}-9\right) / s & 0 & 0 \\
0 & 0 & 1 / \sqrt{\nu} & 0 & 0 & 0 \\
\left(\lambda^{2}+9\right) / s & \left(-\omega^{2}+9\right) / t & 0 & \left(\lambda^{2}+9\right) / s & 0 & 0 \\
\left(\lambda^{3}-7 \lambda\right) / s & 0 & 0 & \left(-\lambda^{3}+7 \lambda\right) / s & \left(-\omega^{3}-7 \omega\right) / t & 0 \\
0 & 0 & 0 & 0 & 0 & \sqrt{v}
\end{array}\right),
$$

sets the quadratic terms (11) into the real normal form

$$
\mathcal{H}_{2}=\lambda q_{x} p_{x}+\frac{\omega}{2}\left(q_{y}^{2}+p_{y}^{2}\right)+\frac{v}{2}\left(q_{z}^{2}+p_{z}^{2}\right)
$$

where $\lambda, \omega$ and $v$ are the positive quantities defined in (7). In (12), we have kept the same notation for the variables after the coordinate change, which is defined by the eigenvectors of $J \nabla \mathcal{H}_{2}$ scaled by

$$
s=\sqrt{2 \lambda\left(16 \lambda^{2}-72\right)}, \quad t=\sqrt{\omega\left(16 \omega^{2}+72\right)},
$$

The complex normal form for $\mathcal{H}_{2}$ simplifies the resolution of the homological equations required for the determination of the generating functions used in the computation of the centre manifold. The complexification is given by

$$
\begin{aligned}
& q_{x}=q_{1}, \quad q_{y}=\frac{q_{2}+i p_{2}}{\sqrt{2}}, \quad q_{z}=\frac{q_{3}+i p_{3}}{\sqrt{2}}, \\
& p_{x}=p_{1}, \quad p_{y}=\frac{i q_{2}+p_{2}}{\sqrt{2}}, \quad p_{z}=\frac{i q_{3}+p_{3}}{\sqrt{2}} .
\end{aligned}
$$

In this way, we get

$$
\mathcal{H}_{2}=\lambda q_{1} p_{1}+i \omega q_{2} p_{2}+i v q_{3} p_{3}
$$

Denoting by $\mathbf{q}=\left(q_{1}, q_{2}, q_{3}\right)^{T}$ and $\mathbf{p}=\left(p_{1}, p_{2}, p_{3}\right)^{T}$, the Hamiltonian can be expanded as

$$
\mathcal{H}=\mathcal{H}_{2}(\mathbf{q}, \mathbf{p})+\sum_{k \geq 3} \mathcal{H}_{k}(\mathbf{q}, \mathbf{p}),
$$

where $\mathcal{H}_{2}$ is given by (14) and $\mathcal{H}_{k}$ is a homogeneous polynomial of degree $k$ in the variables $q_{1}, q_{2}, \ldots, p_{3}$. If we only consider $\mathcal{H}_{2}$, the instability is associated with the term $\lambda q_{1} p_{1}$. For this linear approximation of the equations, the central part can be obtained by setting $q_{1}=p_{1}=0$. If we want the trajectory to remain tangent 
Table 1. Coefficients of the quadratic and cubic terms of the reduced Hamiltonian. The exponents $k_{1}, k_{2}, k_{3}, k_{4}, k_{5}, k_{6}$ correspond to the (real) variables $q_{1}, p_{1}, q_{2}, p_{2}, q_{3}$, $p_{3}$, respectively

\begin{tabular}{ccccccc}
\hline$k_{1}$ & $k_{2}$ & $k_{3}$ & $k_{4}$ & $k_{5}$ & $k_{6}$ & $h_{k}$ \\
\hline 1 & 1 & 0 & 0 & 0 & 0 & $2.508286790247315 e+00$ \\
0 & 0 & 2 & 0 & 0 & 0 & $1.035797111181671 e+00$ \\
0 & 0 & 0 & 2 & 0 & 0 & $1.035797111181671 e+00$ \\
0 & 0 & 0 & 0 & 2 & 0 & $1.000000000000000 e+00$ \\
0 & 0 & 0 & 0 & 0 & 2 & $1.000000000000000 e+00$ \\
3 & 0 & 0 & 0 & 0 & 0 & $-1.236056596683260 e-01$ \\
2 & 1 & 0 & 0 & 0 & 0 & $7.549835794876536 e-01$ \\
1 & 2 & 0 & 0 & 0 & 0 & $-7.549835794876536 e-01$ \\
0 & 3 & 0 & 0 & 0 & 0 & $1.236056596683260 e-01$ \\
2 & 0 & 1 & 0 & 0 & 0 & $6.621611364003422 e-01$ \\
0 & 2 & 1 & 0 & 0 & 0 & $-6.621611364003422 e-01$ \\
2 & 0 & 0 & 1 & 0 & 0 & $-3.265783429459123 e-01$ \\
1 & 1 & 0 & 1 & 0 & 0 & $8.760389554987598 e-01$ \\
0 & 2 & 0 & 1 & 0 & 0 & $-3.265783429459123 e-01$ \\
0 & 0 & 2 & 1 & 0 & 0 & $6.621611364003423 e-01$ \\
0 & 0 & 0 & 3 & 0 & 0 & $-4.289371278505288 e-02$ \\
0 & 0 & 0 & 1 & 2 & 0 & $5.461014290613933 e-01$ \\
\hline
\end{tabular}

to this space, we need to have $\dot{q}_{1}=\dot{p}_{1}=0$ when, after adding the nonlinear terms, we set $q_{1}=p_{1}=0$. Due to the autonomous character of the Hamiltonian system, we will get the required condition if, for instance, in the series expansion of the Hamiltonian, all the monomials $h_{k} q_{1}^{k_{1}} p_{1}^{k_{2}} q_{2}^{k_{3}} p_{2}^{k_{4}} q_{3}^{k_{5}} p_{3}^{k_{6}}$ with $k_{1}+k_{2}=1$ are such that $h_{k}=0$, where $k=\left(k_{1}, k_{2}, k_{3}, k_{4}, k_{5}, k_{6}\right)$. Another possibility is to remove from the Hamiltonian all the monomials with $k_{1} \neq k_{2}$ (see [17]).

This normal form computation has been carried out computing, at each order of the Hamiltonian $\mathcal{H}_{k}$, the generating function $G_{k}$ (which is also a homogeneous polynomial of the degree $k$ ) that removes the suitable terms of the Hamiltonian according to the first criterion mentioned above. A sample of the results obtained after the reduction and realification, using (13), is given in table 1 .

\subsection{Behaviour of the Poincaré map in the centre manifold of $\mathrm{L}_{1}$}

The phase space in the centre manifold is 4-dimensional. To describe the dynamics we fix a Poincaré section, $q_{3}=0, p_{3}>0$, and we use as a parameter the energy level $h$ of the reduced Hamiltonian (note that $h=0$ for the libration point). With these two reductions we can get a qualitative description of the phase space, in a neighbourhood of the libration point, if we plot the 2-dimensional sections for several energy levels. This numerical exploration of the Poincare map could not be done without skipping the instability by means of the reduction to the centre manifold. As a drawback, the procedure does not produce explicit solutions, which should be computed by other methods, for instance with Lindstedt-Poincaré procedures.

In figure 1 , we have represented two of these sections with a different qualitative behaviour. The first one corresponds to $h=0.2$ and the second to $h=0.6$. Both 

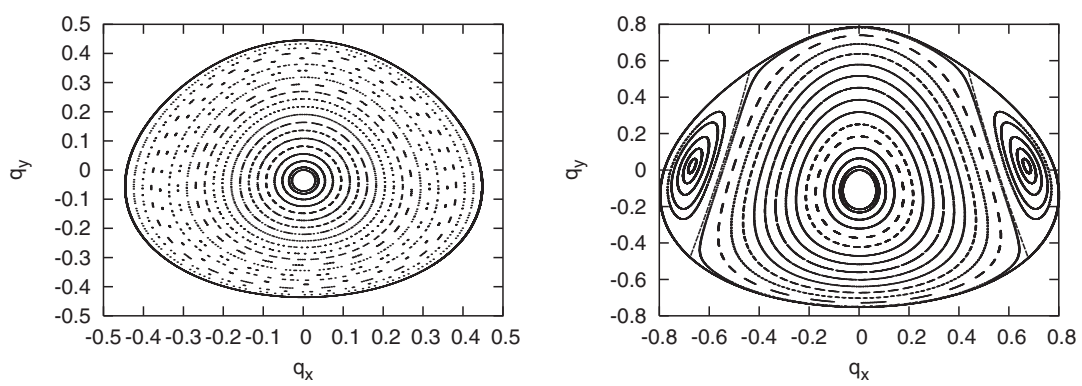

Figure 1. Poincaré map representations of the centre manifold of $L_{1}$ for $h=0.2$ (left) and $h=0.6$ (right). For both figures, the centre manifold coordinates $q_{2}$ and $p_{2}$ have been represented and the surface of section is defined by $q_{3}=0, p_{3}>0$.

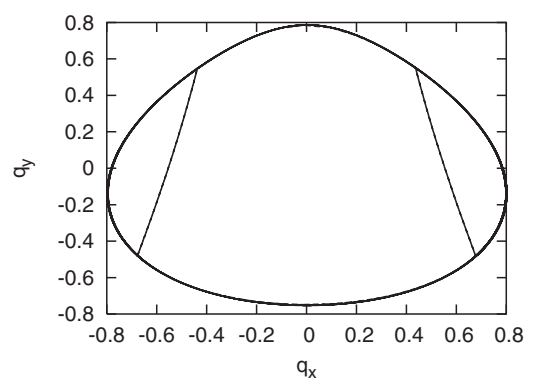

Figure 2. For $h=0.6,\left(q_{2}, p_{2}\right)$-projection of the intersections with the surface of section of the stable and unstable manifolds of the planar Lyapunov orbit in centre manifold coordinates.

have some common features: the outer curve in both plots is the planar Lyapunov periodic orbit while at the middle $\left(q_{2}=0\right)$ there is the fixed point of the Poincare map associated to the vertical Lyapunov periodic orbit. The fixed point associated to the vertical periodic orbit is surrounded, in both cases, by invariant curves of the so-called Lissajous orbits associated to 2D tori around the vertical periodic orbits. The resonant zones between the invariant curves are very hard to detect, at least for these energy values.

On continuing from $h=0.2$ to $h=0.6$, the planar Lyapunov orbit, reduced to the centre manifold, becomes hyperbolic. At the critical value where the stability changes, a bifurcation takes place and two new families of periodic orbits are born, they are the 3D halo orbits. At the same time, associated to the two new real eigenvalues of the monodromy matrix, there appear stable and unstable manifolds. These invariant manifolds act as separatrices of two different kinds of quasi-periodic motion: the Lissajous orbits already mentioned and the so-called quasi-halo orbits, which are also 2D tori but around the two symmetric families of periodic halo orbits. The computation of these manifolds must be done using the reduced Hamiltonian, since one of the hyperbolic eigenvalues (the one that has been removed with the reduction) is about 1000 larger than the new hyperbolic one that has appeared after the destabilization of the Lyapunov orbit.

In figure 2, we have represented the intersections of the stable and unstable manifolds with the surface of section $q_{3}=0, p_{3}>0$ for $h=0.6$. Both manifolds, 

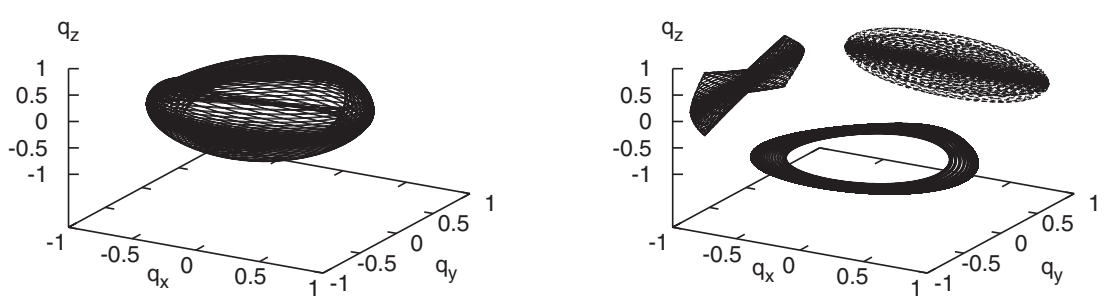

Figure 3. 3D representation (left) in the $\left(q_{2}, p_{2}, q_{3}\right)$-space and coordinate projections (right) of an orbit of the stable manifold of the planar Lyapunov orbit with $h=0.6$ (centre manifold coordinates). The intersections of this orbit with the surface of section $q_{3}=0$ are displayed in figure 2 .

when far from the Lyapunov planar orbit, look like two segments joining two couples of symmetrical points of the periodic orbit. In fact, the stable and unstable manifolds almost coincide and it s very hard to distinguish one from the other. An orbit of one of these invariant manifolds is shown in figure 3. These solutions can be useful for practical applications, since they perform a transition from an almost planar motion (close to the Lyapunov orbit) to an inclined one (close to the quasi-halo orbits) without any $\Delta v$. Unfortunately, this natural transition is slow.

For larger values of the energy, the semi-numerical approximation of the centre manifold is no longer good enough. In the next sections we will show how the periodic and quasi-periodic solutions can be continued by means of direct numerical methods.

\section{Periodic orbits}

The numerical computation of periodic orbits of Hamiltonian systems is a wellknown topic and we will not go into the details, which the reader can find, for instance, in [18]. For the present study we have used the same predictor-corrector procedure, based on the integration of the variational equations, that was used in [19] for the RTBP.

Let $\phi_{t}(\mathbf{x})$ be the flow associated to the Hamiltonian $\mathcal{H}$ of Hill's problem. The normal behaviour of a $T$-periodic orbit through $\mathbf{x}_{0}$ is studied in terms of the time- $T$ flow around $\mathbf{x}_{0}$, whose linear approximation is given by the monodromy matrix $M=D \phi_{T}\left(\mathbf{x}_{0}\right)$ of the periodic orbit. Since the eigenvalues of $M$ are $\left\{1,1, \lambda_{1}, \lambda_{1}^{-1}, \lambda_{2}, \lambda_{2}^{-1}\right\}$, the stability parameters of the periodic orbit can be defined as $s_{j}=\lambda_{j}+\lambda_{j}^{-1}$, for $j=1,2$. They can be of one of the following kinds:

- Hyperbolic: $s_{j} \in \mathbb{R},\left|s_{j}\right|>2$. It is equivalent to $\lambda_{j} \in \mathbb{R} \backslash\{-1,1\}$.

- Elliptic: $s_{j} \in \mathbb{R},\left|s_{j}\right|<2$. It is equivalent to $\lambda_{j}=e^{i \rho}$ with $\rho \in \mathbb{R}$ (if $\left|s_{j}\right|=2$, then it is said to be 'parabolic').

- Complex unstable: $s_{j} \in \mathbb{C} \backslash \mathbb{R}$. It is equivalent to $\lambda_{j} \in \mathbb{C} \backslash \mathbb{R},\left|\lambda_{j}\right| \neq 1$.

Special attention will be devoted to periodic orbits with elliptic stability parameters. If $s_{j}$ is elliptic, the $\left\{\lambda_{j}, \lambda_{j}^{-1}\right\}$-eigenplane of $M$ through $\mathbf{x}_{0}$ is foliated (in the linear approximation) by invariant curves of the restriction of the linearization of $\phi_{T}$ (that is, the map $\mathbf{x} \rightarrow \mathbf{x}_{0}+M\left(\mathbf{x}-\mathbf{x}_{0}\right)$ ), which have rotation number $\rho$. For the full system, some of these invariant curves persist and give rise to $2 \mathrm{D}$ tori, as will be shown in section 6 . 

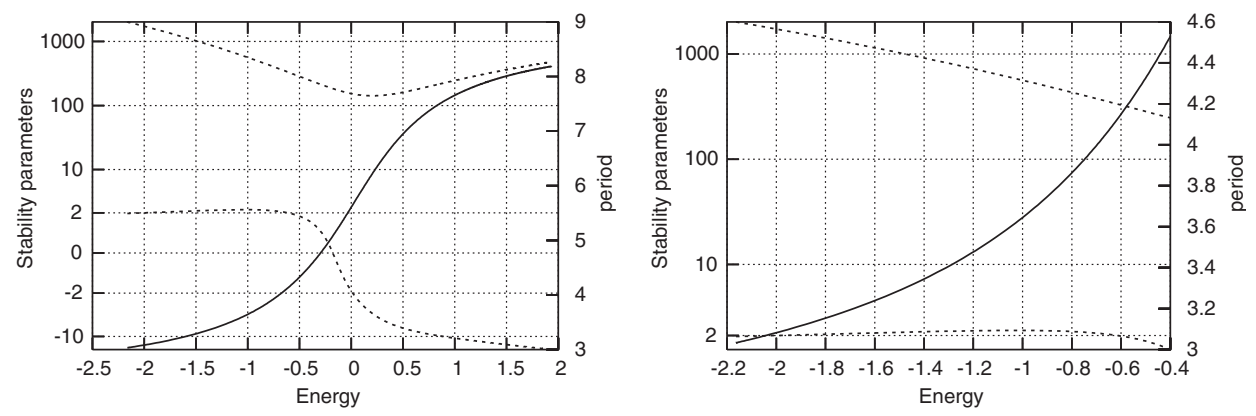

Figure 4. Characteristic curve (solid line) and stability parameters (dashed lines) of the planar Lyapunov family of periodic orbits. The right-hand figure is a magnification of the region of orbits with elliptic normal behaviour.
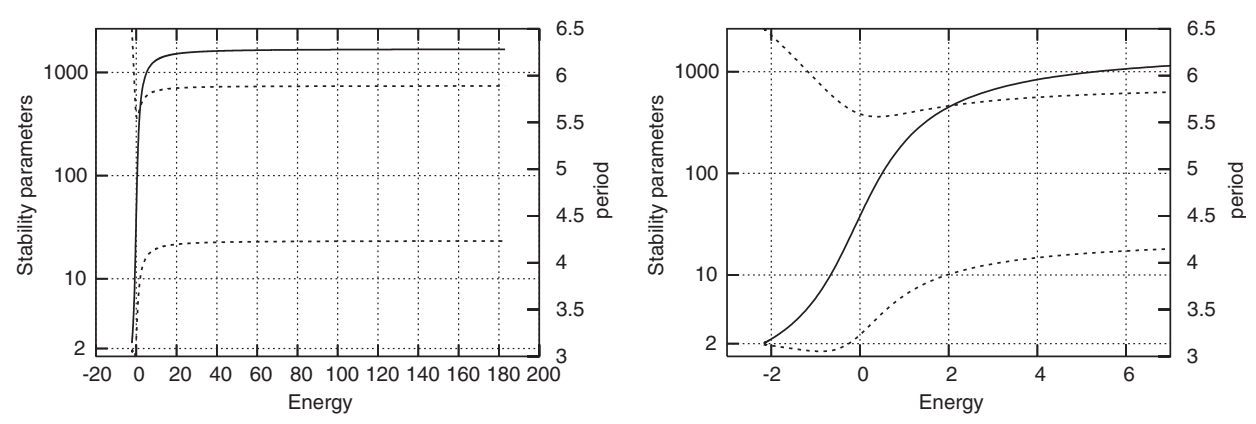

Figure 5. Characteristic curve (solid line) and stability parameters (dashed lines) of the vertical Lyapunov family of periodic orbits. The right-hand figure is a magnification of the region of orbits with elliptic normal behaviour.

According to Lyapunov's centre theorem (see [20]) and the numerical values given in (7), there are two families of periodic orbits emanating from the equilibrium point $L_{1}$ : the vertical Lyapunov family and the planar Lyapunov family. Both families are born at the energy level of $L_{1}: \mathcal{H}\left(L_{1}\right)=-2 \cdot 3^{-2 / 3}-3^{1 / 3}=-2.40374928 \ldots$

In figures 4 and 5 , we have represented the characteristic curves (energy vs. period) and the stability curves (energy vs. $s_{i}$ ) for both families. Since the range of the stability parameters is very large, we have plotted $2 \operatorname{arcsinh}\left(s_{i}\right) / \operatorname{arcsinh}(2)$ instead of $s_{i}$.

Following [21], we can detect and compute the 3D families of periodic orbits that bifurcate from the Lyapunov planar family. The results obtained are the following:

(1) At the first bifurcation, which is a Type A bifurcation in Hénon's terminology, there appear two symmetrical families of periodic orbits with respect to the $q_{z}=0$ plane. The bifurcation takes place when $\mathcal{H}=-2.00266$, and the families are known as north and south class halo families, respectively. Their characteristic and stability curves are given in figure 6 .

(2) The second bifurcation is of Type B, and there appear two families of periodic orbits. The orbits are symmetrical with respect to the $q_{x}$-axis and each orbit in one family has a symmetrical orbit, with respect to the $q_{z}=0$ plane, 


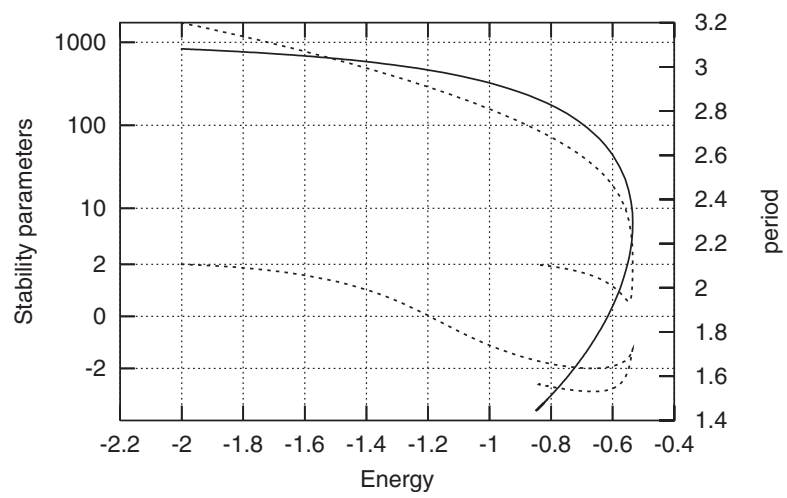

Figure 6. Characteristic curve (solid line) and stability parameters (dashed lines) of the halo family of periodic orbits.
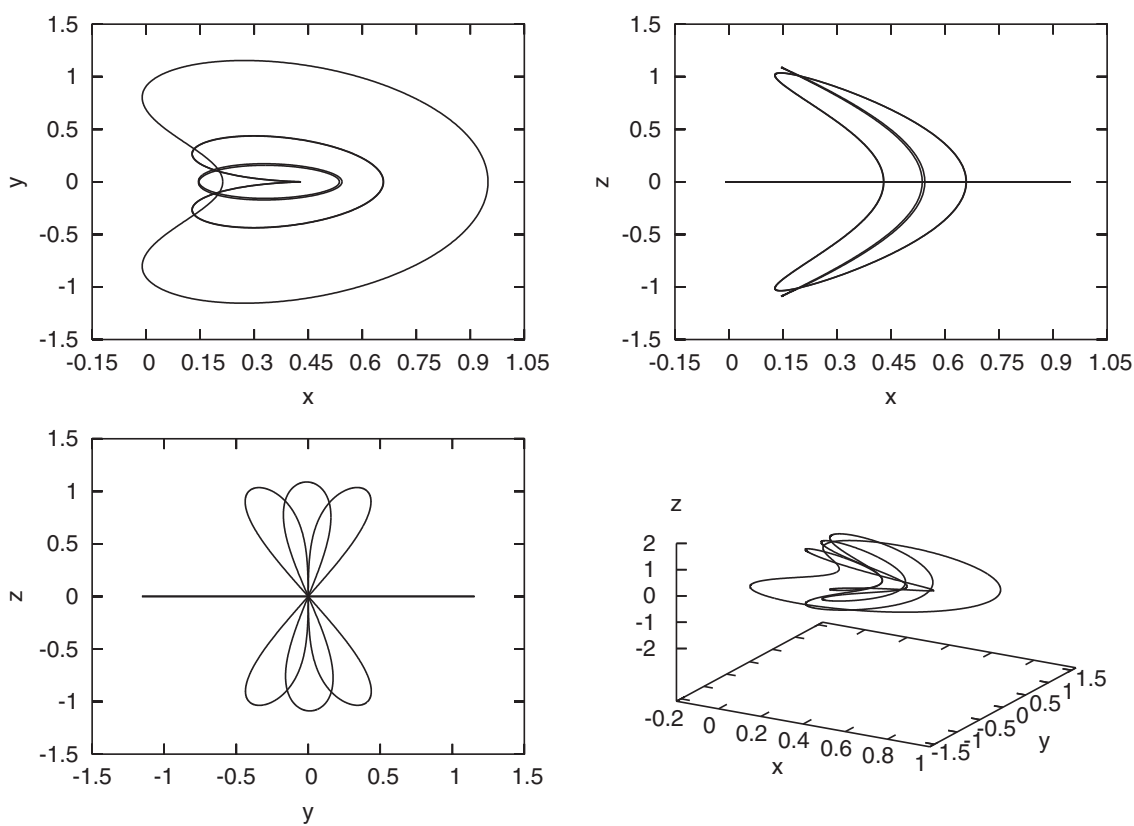

Figure 7. Coordinate projections and $3 \mathrm{D}$ representation of some orbits of the two lane bridge of periodic orbits connecting the planar Lyapunov family of periodic orbits with the vertical one.

in the other, as is shown in figure 7. The orbits of both families form a two lane bridge, connecting the planar Lyapunov family with the vertical one. Their characteristic and stability curves are given in figure 8.

Along the family of halo orbits, there appear three bifurcations that will be relevant in the qualitative description of the phase space. For the first one, which happens at $\mathcal{H}=-0.97607$, the small stability parameter is equal to $2 \cos (2 \pi / 3)$, so it gives rise to two period-tripling families. One of the bifurcated families has 


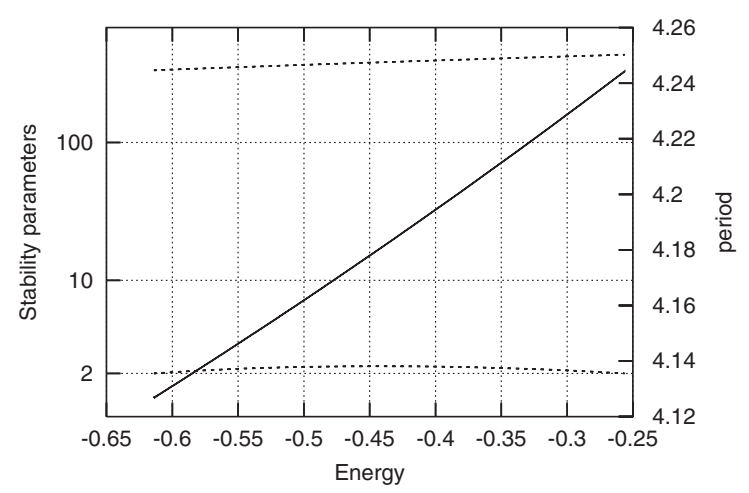

Figure 8. Characteristic curve (solid line) and stability parameters (dashed lines) of the bridge of periodic orbits connecting the planar Lyapunov family of periodic orbits with the vertical one.

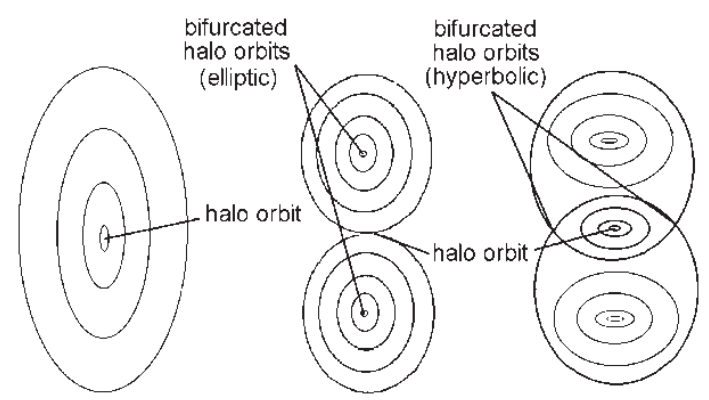

Figure 9. Qualitative representation of the two period-doubling bifurcations of the $L_{1}$ halo family.

an elliptic stability parameter, whereas the other has both stability parameters hyperbolic. As for the second and third bifurcations, they are period doubling, since they correspond to a value of the small stability parameter equal to $2 \cos (2 \pi / 2)=-2$. This happens at energies $\mathcal{H}=-0.67004$ and -0.66376 . At the first bifurcation the bifurcated family has one elliptic stability parameter, while the second bifurcation has both stability parameters hyperbolic. These two bifurcations are represented qualitatively in figure 9.

\section{Stable/unstable manifolds of the centre manifold}

The aim of this section is the computation of the stable and unstable manifolds of the centre manifolds of $L_{1}$ and $L_{2}$. In the next section, we will study their homoclinic and heteroclinic connections.

Assume that we have already obtained, for instance, the stable manifold of some central trajectory around $L_{1}$. Making use of the symmetries, its unstable manifold is easily found by means of

$$
\left(q_{x}(t), q_{y}(t), q_{z}(t)\right) \rightarrow\left(q_{x}(-t),-q_{y}(-t), q_{z}(-t)\right),
$$


and the stable/unstable manifolds of the symmetric trajectory around $L_{2}$, can be also obtained, without any further computation, using now

$$
\left(q_{x}(t), q_{y}(t), q_{z}(t)\right) \rightarrow\left(-q_{x}(-t), q_{y}(-t), q_{z}(-t)\right) .
$$

So, the determination of all the manifolds is reduced to the computation of just one manifold for one of the equilibrium points.

In order to compute one of these manifolds, we have made use of the reduced Hamiltonian of the problem. Recall that when we removed the monomials $q_{1}^{k_{1}} p_{1}^{k_{2}} q_{2}^{k_{3}} p_{2}^{k_{4}} q_{3}^{k_{5}} p_{3}^{k_{6}}$ with $k_{1}+k_{2}=1$, we obtained the reduction to the centre manifold. Nevertheless, in the implementation of the procedure we have computed all the coefficients $h_{k}$ and then set $h_{k}=0$ for those with $k_{1}+k_{2}=1$. Now, if for one central trajectory, determined by the values of $q_{2}, p_{2}, q_{3}, p_{3}$, we set $q_{1} \neq 0$ or $p_{1} \neq 0$ we get its stable or unstable manifolds. The two branches of each manifold are determined taking positive and negative values for these two coordinates. This representation of both manifolds is much more accurate than the one that can be obtained using only the linear approach of the flow (which is obtained taking only the quadratic terms $\mathcal{H}_{2}$ of the reduced Hamiltonian). This allows the use of moderate values for $q_{1}$ and $p_{1}$; for our computations we have used values between 0.1 and 0.01 .

Since we are interested in the computation of heteroclinic trajectories between the centre manifolds of $L_{1}$ and $L_{2}$, we have to match an orbit of the unstable manifold of a libration orbit around one point with another orbit in the stable manifold of a libration orbit around the other point. As these orbits go from the vicinity of $L_{1}$, which has $q_{x}>0$, to the vicinity of $L_{2}$, with $q_{x}<0$, the section that has been used to detect the connections is the plane $q_{x}=0$, that is, the plane orthogonal to the $q_{x}$-axis at the point $q_{x}=0$.

For the globalization of the invariant manifolds, in principle, we should have to take into account the two branches (positive and negative) of each manifold. Nevertheless, as is shown in figure 10 for the Lyapunov planar periodic orbits, one of the branches is always in the outer region of the zero velocity curve, so with them we cannot get any kind of heteroclinic connection. The orbits on these branches get close to the two asymptotes $q_{x}= \pm \sqrt{C / 3}$ of the zero velocity curves, that can be obtained from (6). This behaviour is common to all the libration point orbits.
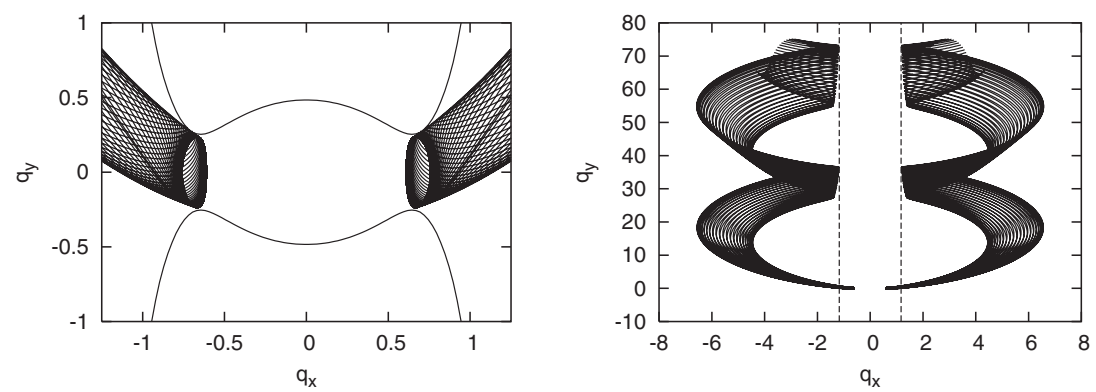

Figure 10. Second branch of the stable manifold of the Lyapunov planar periodic orbits around $L_{1}$ and $L_{2}$. The left-hand figure is a magnification of the right-hand one and in it we have represented the zero velocity curve of the energy level. 

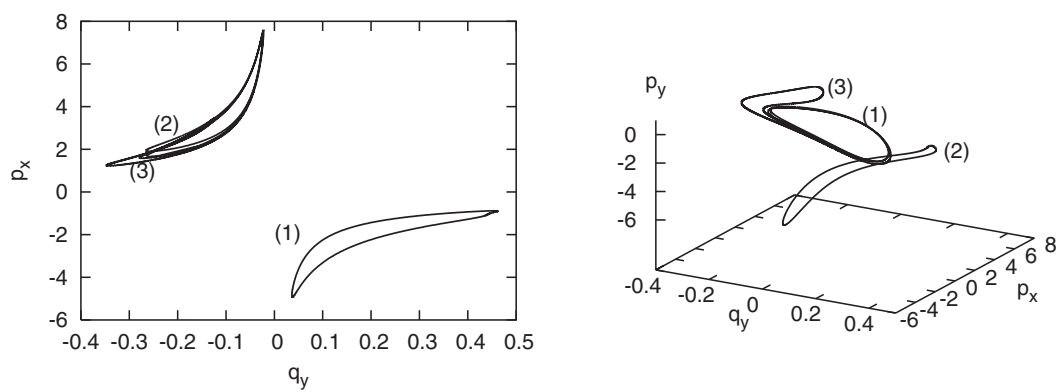

Figure 11. First three intersections with $q_{x}=0$ of the stable manifold of the planar Lyapunov periodic orbit with $h=0.2$. The left-hand plot corresponds to the $\left(q_{y}, p_{x}\right)$ projection and the right-hand one to the $\left(q_{y}, p_{x}, p_{y}\right)$-projection.
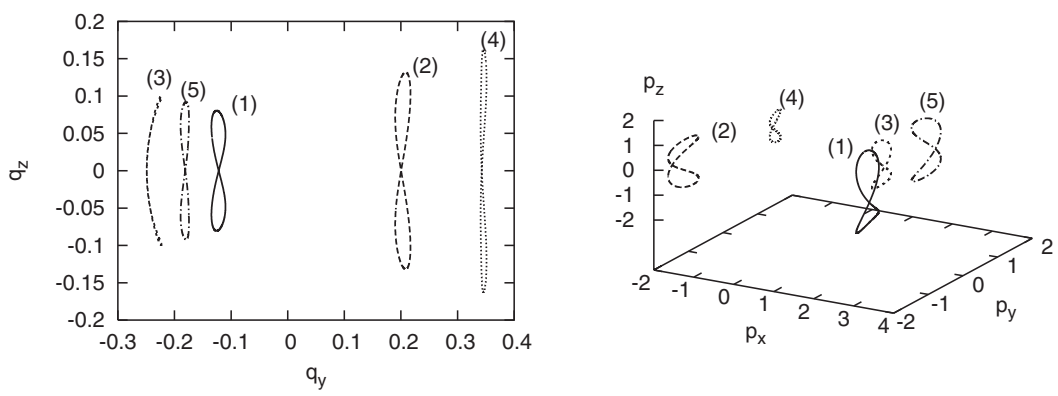

Figure 12. First five intersections with $q_{x}=0$ of the stable manifold of the vertical Lyapunov periodic orbit with $h=0.2$. The left-hand plot corresponds to the $\left(q_{y}, q_{z}\right)$ projection and the right-hand one to the $\left(p_{x}, p_{y}, p_{z}\right)$-projection.

In figures 11 and 12, we show the projections of the first intersections of the stable manifold of the planar and vertical Lyapunov periodic orbits of energy $h=0.2$ with this surface of section. The qualitative results for other values of the energy are similar. As the number of intersections increases, the trace of the manifold with the section has more and more foldings, which must be computed carefully. The different foldings tend to get close to the low-order intersections, as can be seen in figure 11 for the third intersection, which is already rather close to the first.

The projections displayed in figure 13 correspond to the first three intersections of the stable manifold of all the central orbits around $L_{1}$ for $h=0.2$ and $h=0.6$. Recall that for these two values of the energy, the Poincare map has different qualitative behaviour (see figure 4). As has already been mentioned, for these numerical computations, the regularized equations must be used, since many trajectories have close approaches to the origin, in which equations (4) have a singularity.

\section{Homoclinic and heteroclinic connections}

With the stable and unstable manifolds, and their intersections with the plane $q_{x}=0$ obtained in the preceding section, we are able to compute different kinds of 

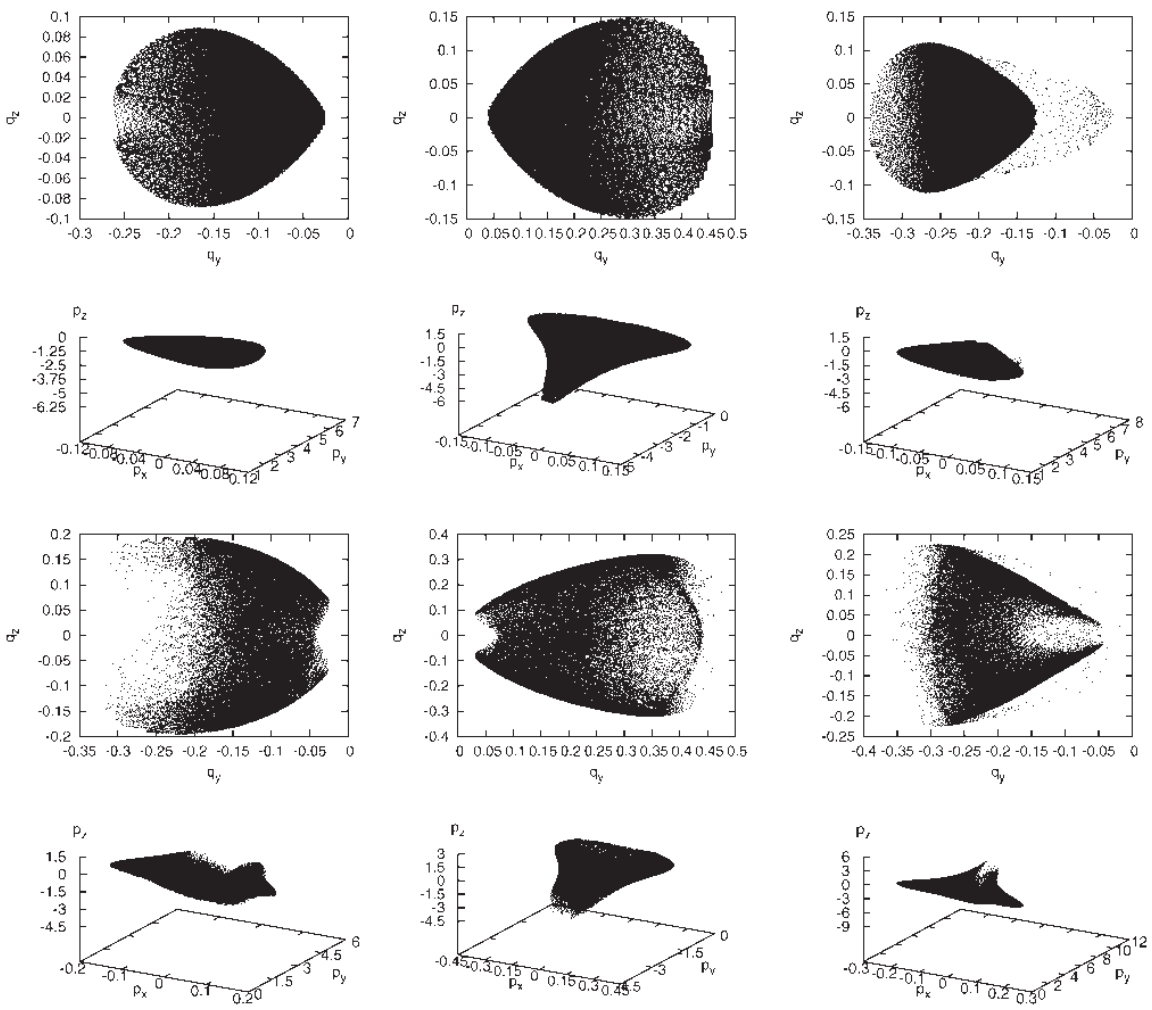

Figure 13. First three intersections with $q_{x}=0$ of the stable manifold of the centre manifold of $L_{1}$ for $h=0.2$ (first two rows) and $h=0.6$ (last two rows). The three plots of the first and third row correspond to the $\left(q_{y}, q_{z}\right)$-projections of the first, second and third intersections. The plots of the second and last row are the corresponding $\left(p_{x}, p_{y}, p_{z}\right)$-projections.

homoclinic and heteroclinic orbits. They will be classified according to the number of times they cross the $q_{x}=0$ plane (see figure 14). The $q$ th intersection of $W_{L_{1}}^{s}$ (p.o.) with $q_{x}=0$ will be labelled $\Gamma_{L}^{s, q}(p . o$.$) , where p.o. denotes a certain periodic orbit.$ Similarly, we will denote by $\Gamma_{L_{2}}^{u, p}\left(W_{h}^{c}\right)$ the $p$ th intersection with $q_{x}=0$ of the centre manifold of $L_{2}$ at the energy level $h, W_{L_{2}}^{u}\left(W_{h}^{c}\right)$.

With this notation, the low-order homoclinic connections of a periodic orbit will correspond to

$$
\Gamma_{L_{1}}^{u, 1}(\text { p.o. }) \cap \Gamma_{L_{1}}^{s, 2}(\text { p.o. }), \quad \Gamma_{L_{1}}^{u, 1}(\text { p.o. }) \cap \Gamma_{L_{1}}^{s, 4}(\text { p.o. })=\Gamma_{L_{1}}^{u, 2}(\text { p.o. }) \cap \Gamma_{L_{1}}^{s, 3}(\text { p.o. }),
$$

and the heteroclinic connections to

$$
\begin{gathered}
\Gamma_{L_{1}}^{u, 1}(\text { p.o. }) \cap \Gamma_{L_{2}}^{s, 1}(\text { p.o. }), \quad \Gamma_{L_{1}}^{u, 1}(\text { p.o. }) \cap \Gamma_{L_{2}}^{s, 3}(\text { p.o. })=\Gamma_{L_{1}}^{u, 2}(\text { p.o. }) \cap \Gamma_{L_{2}}^{s, 2}(\text { p.o. }), \\
\Gamma_{L_{1}}^{u, 1}(\text { p.o. }) \cap \Gamma_{L_{2}}^{s, 5}(\text { p.o. })=\Gamma_{L_{1}}^{u, 2}(\text { p.o. }) \cap \Gamma_{L_{2}}^{s, 4}(\text { p.o. })=\Gamma_{L_{1}}^{u, 3}(\text { p.o. }) \cap \Gamma_{L_{2}}^{s, 3}(\text { p.o. }) .
\end{gathered}
$$

In what follows, we will show the results for the energy level $h=0.2$, which are qualitatively identical to the ones obtained for values of $h$ up to the one 


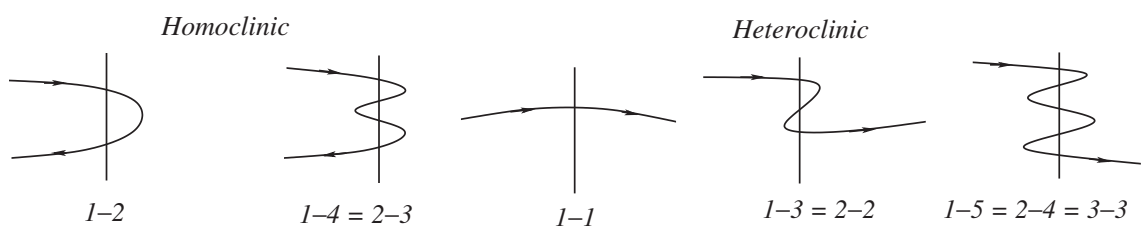

Figure 14. According to the number of intersections with the section $q_{x}=0$, there are different kinds of homoclinic and heteroclinic orbits.
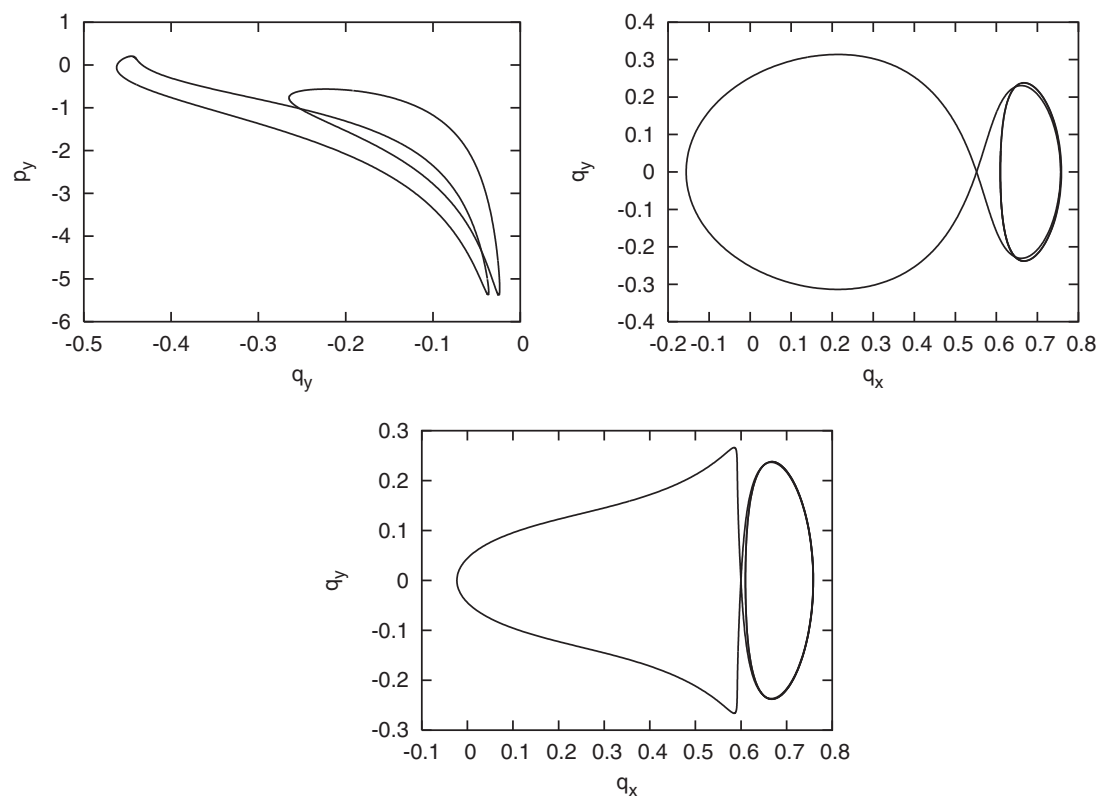

Figure 15. $\left(q_{y}, p_{y}\right)$-projection of the intersections of the stable manifold (first cut with the section $q_{x}=0$ ) with the unstable manifold (second intersection) of the planar Lyapunov orbit with $h=0.2$. The two homoclinic planar orbits are also displayed in the figure, $\left(q_{x}, q_{y}\right)$-projection.

corresponding to the bifurcation of the halo-periodic orbits. The results for values of $h$ after the bifurcation of the halos will appear in [22].

If we consider the Lyapunov planar periodic orbit, the two sets $\Gamma_{L_{1}}^{u, 1}($ p.p.o. $) \cap$ $\Gamma_{L_{1}}^{s, 2}($ p.p.o. $)$ and $\Gamma_{L_{1}}^{u, 2}($ p.p.o. $) \cap \Gamma_{L_{1}}^{s, 3}($ p.p.o. $)$ are shown in figures 15 and 16 . From them, it can be seen that (at this energy level) $\Gamma_{L_{1}}^{u, 1}$ (p.p.o.) $\cap \Gamma_{L_{1}}^{\text {s,2 }}$ (p.p.o.) has two points, while $\Gamma_{L_{1}}^{u, 2}$ (p.p.o.) $\cap \Gamma_{L_{1}}^{s, 3}$ (p.p.o.) has five. The corresponding planar homoclinic orbits are also displayed in both figures. For the vertical periodic orbit we have not been able to find any homoclinic connection for any value of the energy in the range explored. If fact, the intersections of the $\Gamma_{L_{1}}^{u / s, p}($ v.p.o.) sets are empty for all the values of $p \leq 6$.

When moving to a Lissajous orbit close to the planar Lyapunov periodic orbit, it seems reasonable to find homoclinic connections near to those found for the periodic orbit, while if the Lissajous orbit is close to the vertical periodic orbit, the set of 

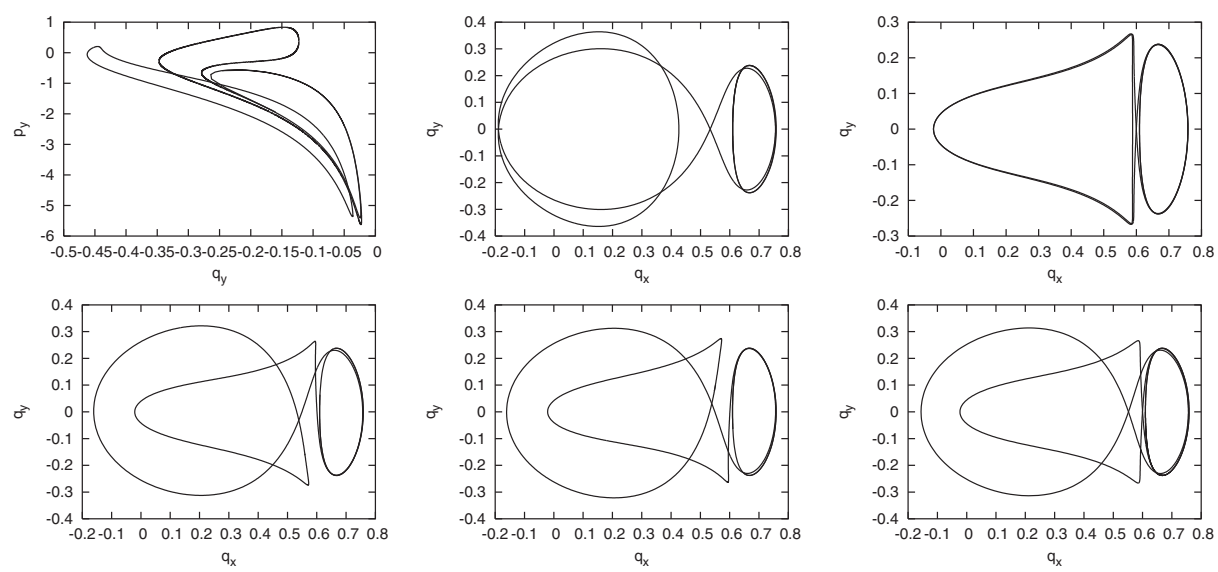

Figure 16. $\left(q_{y}, p_{y}\right)$-projection of the intersections of the unstable manifold (second cut with the section $q_{x}=0$ ) with the stable manifold (third intersection) of the planar Lyapunov orbit with $h=0.2$. The $\left(q_{x}, q_{y}\right)$-projections of the five homoclinic planar orbits are also displayed in the figure.

low-order homoclinic connections probably will be empty. Namely, the planar orbits are periodic and so have invariant manifolds of dimension 2. But, since they are planar, they live in the planar energy manifold, which has dimension 3, and one may expect isolated homoclinic orbits. On the other hand, the Lissajous orbits from 2-dimensional tori have 3-dimensional invariant manifolds but live in a 5-dimensional energy manifold where again one may expect isolated intersections. These are, in fact, the results that have been found. In particular, if we select a Lissajous orbit close to the planar periodic orbit, the $\Gamma_{L_{1}}^{u, 1}($ Liss $) \cap \Gamma_{L_{1}}^{s, 2}($ Liss $)$ set is shown in figure 17. This set has four points that produce the homoclinic orbits displayed in figure 17. These four orbits are closely related to the two homoclinic 1-2 connections of figure 15 . To see this, we have computed the full family of $1-2$ homoclinic connections of the centre manifold of $L_{1}$, for the value of $h$ under consideration. Any of these connections can be determined by the values of $\left(q_{1}, p_{1}\right)$ for the stable/unstable components and $\left(q_{2}, p_{2}, q_{3}, p_{3}\right)$ for the centre manifold coordinates. Since $\left(q_{1}, p_{1}\right)$ and the value of the energy are fixed, each family of homoclinic connections can be represented by a 3-dimensional curve. Now, we can take the points on these curves and follow them under the flow, until they reach the surface of section $q_{3}=0, p_{3}>0$, which was used for the representation of the centre manifold. The results are shown in figure 18 .

Similar results are obtained for the 2-3 homoclinic orbits. Some of them are shown in figure 19 for the same Lissajous orbit as in figure 17.

With respect to heteroclinic connections, it is seen that the set $\Gamma_{L_{1}}^{u, 1}\left(W^{c}\right) \cap \Gamma_{L_{2}}^{s, 1}\left(W^{c}\right)$ is empty. This is clear from figure 20 in which we see that the $\left(q_{z}, p_{y}\right)$-projections of $\Gamma_{L_{1}}^{S, 1}\left(W^{c}\right)$ and $\Gamma_{L_{2}}^{u, 1}\left(W^{c}\right)$ are disjoint.

We have just looked for possible heteroclinic orbits connecting the planar periodic orbits. For $h=0.2$, the set $\Gamma_{L_{1}}^{u, 2}($ p.p.o. $) \cap \Gamma_{L_{2}}^{s, 2}$ (p.p.o.) has only two points, whose

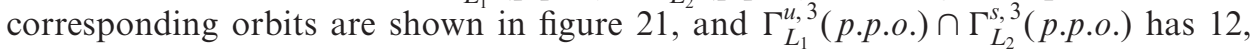
that have been displayed in figure 22. Other heteroclinic connections between Lissajous, halo and quasi-halo orbits can be found in [22]. 

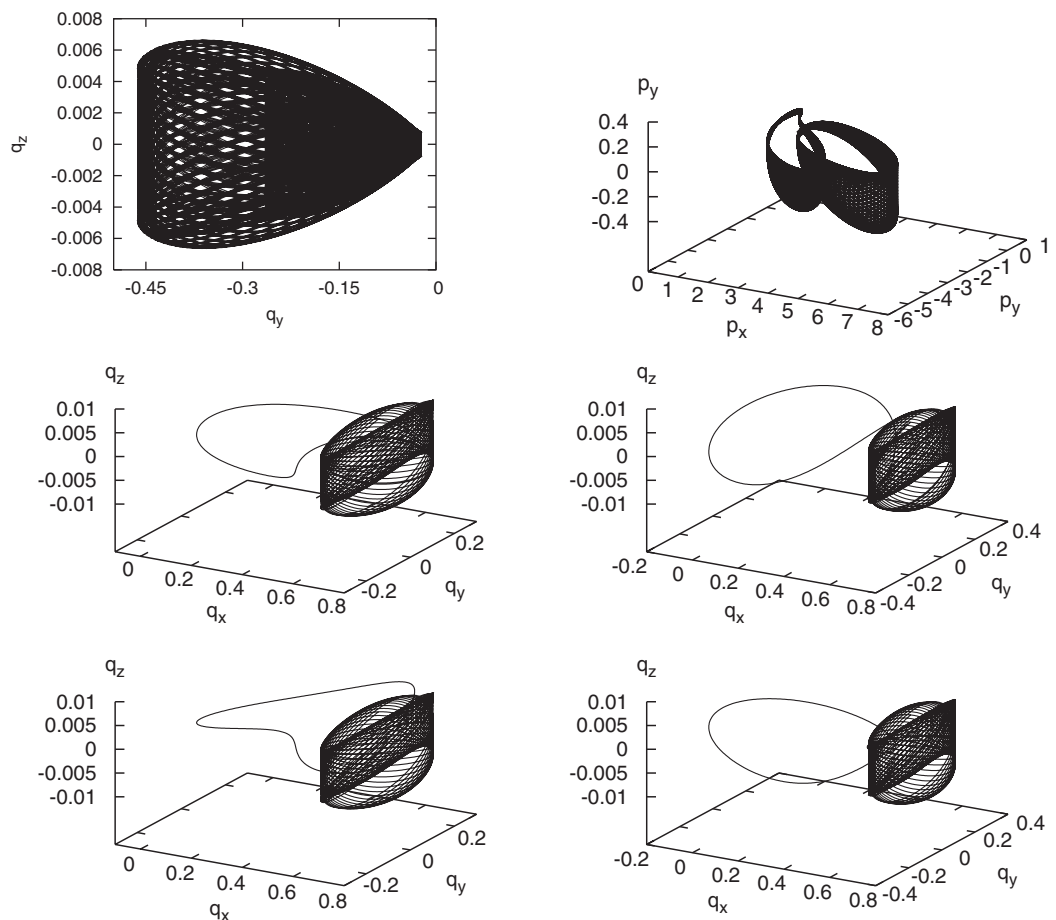

Figure 17. The first line shows $\left(q_{y}, q_{z}\right)$ - and $\left(p_{x}, p_{y}, p_{z}\right)$-projections of the intersections of the stable manifold (first cut with $q_{x}=0$ ) with the unstable manifold (second intersection) of a Lissajous orbit around $L_{1}$ for $h=0.2$. The $\left(q_{x}, q_{y}, q_{z}\right)$ projections of the homoclinic orbits are displayed in the remaining plots.

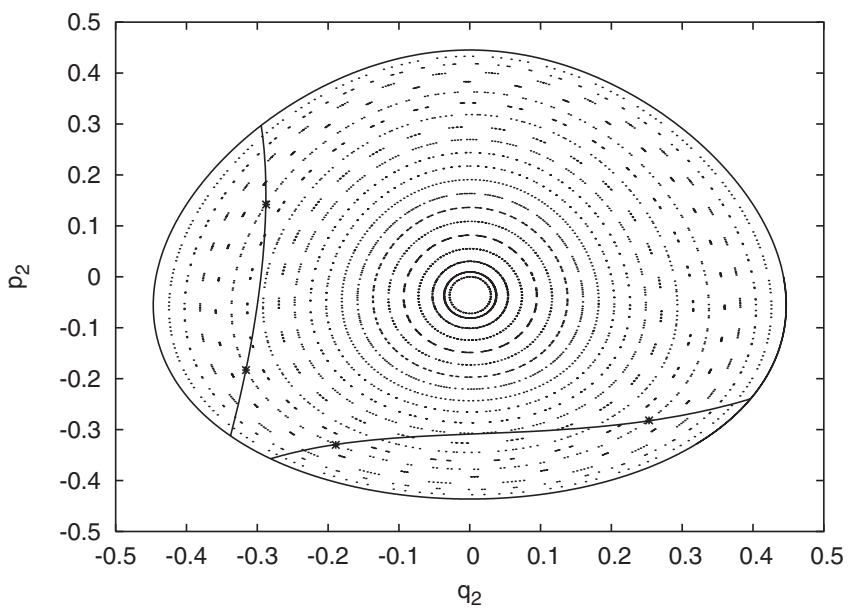

Figure 18. On the Poincaré map representation of the centre manifold for $h=0.2$, we display the two curves of homoclinic 1-2 connections. The four dots, which are on the same Lissajous orbit, correspond to four different homoclinic connections, close to those shown in figure 17 for a nearby orbit. 


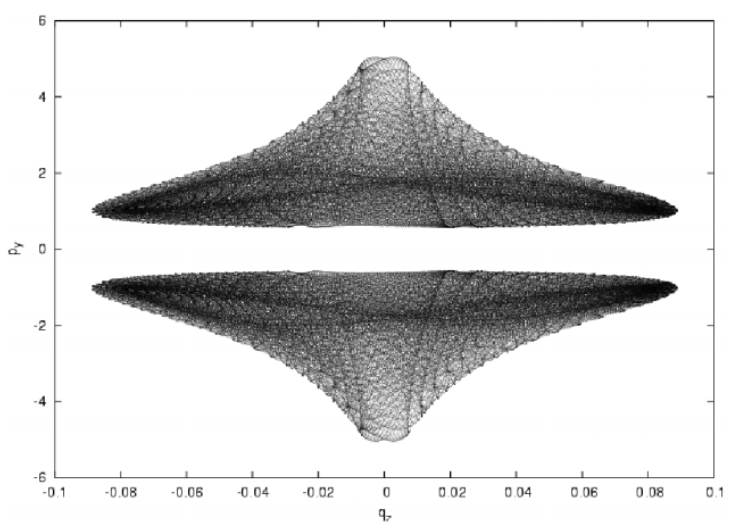

Figure 19. The first line shows $\left(q_{y}, q_{z}\right)$ - and $\left(p_{x}, p_{y}, p_{z}\right)$-projections of the intersections of the stable manifold (second cut with $q_{x}=0$ ) with the unstable manifold (third intersection) of the Lissajous orbit of figure 17 around $L_{1}$ for $h=0.2$. The five heteroclinic connections are also displayed in the figure, $\left(q_{x}, q_{y}, q_{z}\right)$-projection.
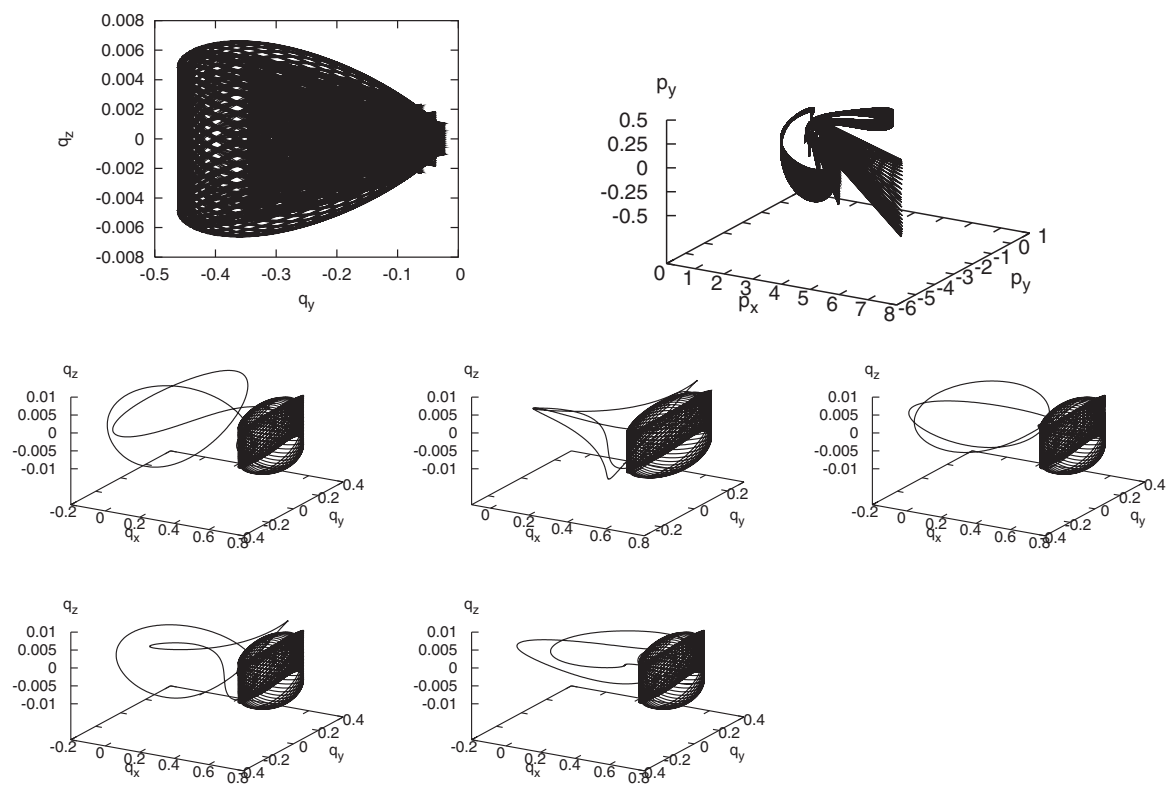

Figure 20. Projections on the $\left(q_{z}, p_{y}\right)$ plane of $\Gamma_{L_{1}}^{s, 1}\left(W^{c}\right)$ and $\Gamma_{L_{2}}^{u, 1}\left(W^{c}\right)$.

As final remark, it must be noted that once we have detected a homoclinic or a heteroclinic orbit, using the symmetries of the problem, we can obtain many other ones. More concretely, if $\left(q_{x}(t), q_{y}(t), q_{z}(t)\right)$ is a homoclinic orbit to a certain Lissajous orbit, then

(i) $s_{1}\left(q_{x}(t), q_{y}(t), q_{z}(t)\right)=\left(q_{x}(-t),-q_{y}(-t), q_{z}(-t)\right)$,

(ii) $s_{3}\left(q_{x}(t), q_{y}(t), q_{z}(t)\right)=\left(q_{x}(t), q_{y}(t),-q_{z}(t)\right)$,

(iii) $s_{3}\left(s_{1}\left(q_{x}(t), q_{y}(t), q_{z}(t)\right)\right)=\left(q_{x}(-t),-q_{y}(-t),-q_{z}(-t)\right)$, 

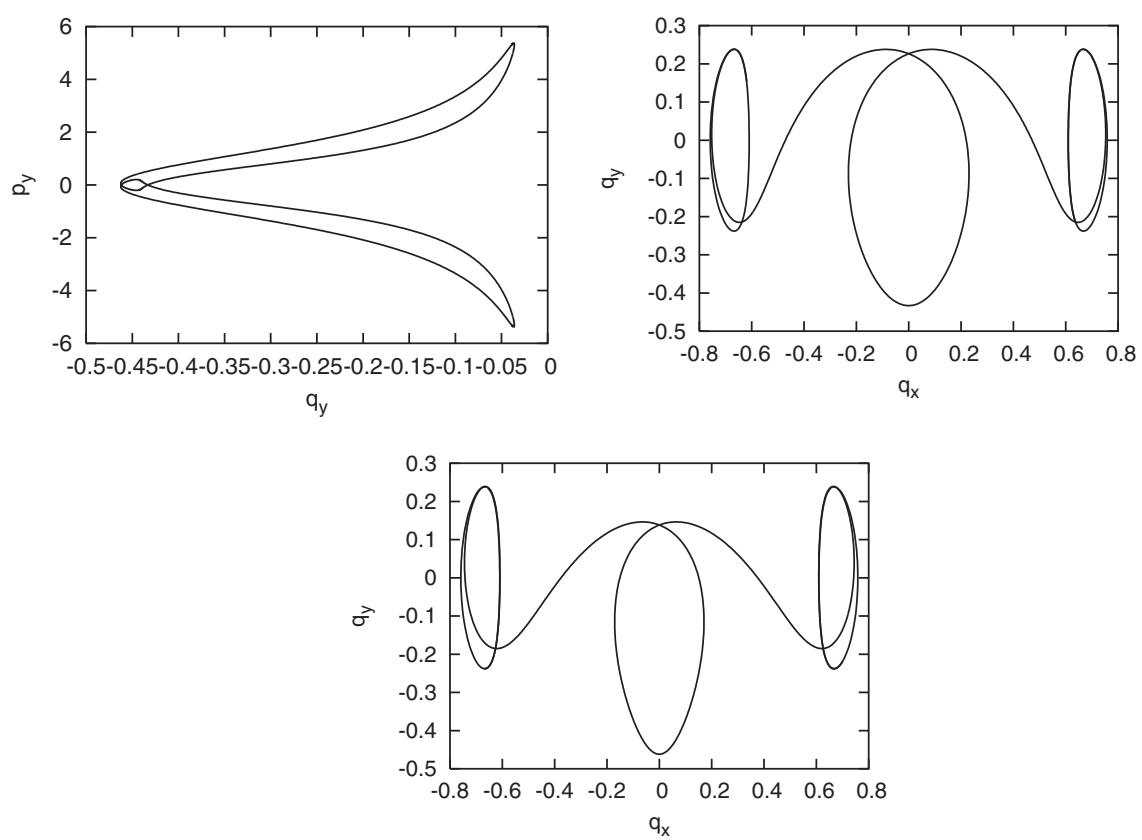

Figure 21. $\left(q_{y}, p_{y}\right)$-projection of the intersections of the stable manifold (second cut with the section $q_{x}=0$ ) around $L_{1}$ with the unstable manifold (second intersection) of the planar Lyapunov orbit around $L_{2}$ for $h=0.2$. The two heteroclinic planar orbits are also displayed in the figure, $\left(q_{x}, q_{y}\right)$-projection.

are also homoclinic orbits of the same Lissajous orbit. If we are interested in the homoclinic orbits of the symmetric Lissajous orbit around the other libration point, we only need to apply the $s_{2}$ symmetry to all the preceeding orbits.

\section{Numerical computations of the centre manifold}

From the normal form computation described in section 2.1, we know that, except from homoclinic and heteroclinic phenomena, the centre manifold of the $L_{1}$ libration point is formed by families of periodic orbits and two-dimensional invariant tori around them. The numerical computation of the relevant families of periodic orbits has been already described in section 3. Here we will briefly introduce the methodology for the numerical computation of invariant tori. After that, we will describe the numerical computation of some families of invariant tori. Finally, we will merge these computations with the ones of periodic orbits of section 3 , in order to extend the Poincare map representations of the flow, computed using the normal form, to values of the energy for which the semi-analytical approximation is no longer valid.

\subsection{Numerical computation of invariant tori}

The procedure used for the refinement and continuation of invariant tori is based on looking for the Fourier series of the parametrization of an invariant curve on a 

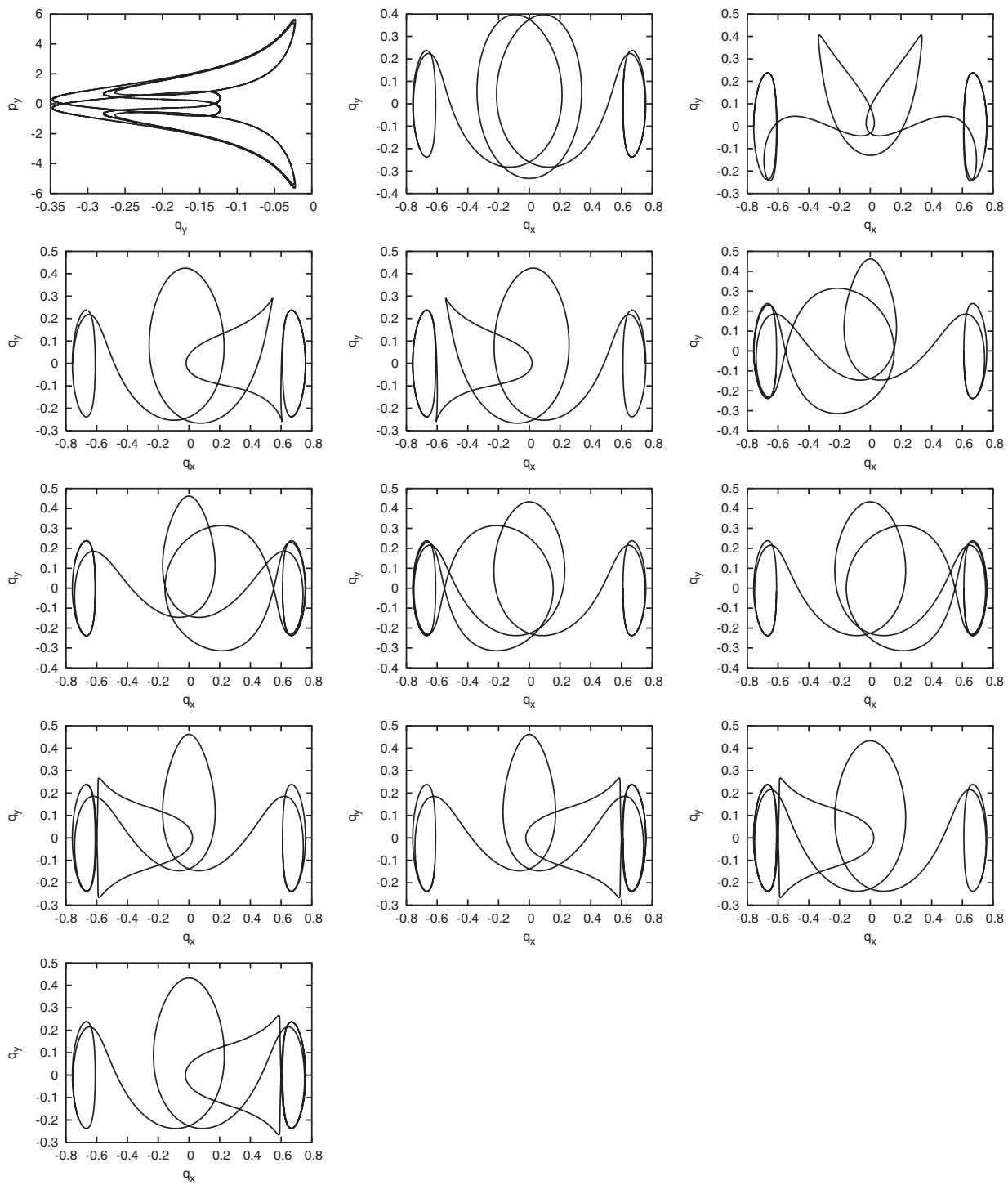

Figure 22. $\left(q_{y}, p_{y}\right)$-projection of the intersections of the stable manifold (third cut with the section $q_{x}=0$ ) around $L_{1}$ with the unstable manifold (third intersection) of the planar Lyapunov orbit around $L_{2}$ for $h=0.2$. The 12 heteroclinic planar orbits are also displayed in the figure, $\left(q_{x}, q_{y}\right)$-projection.

torus, asking numerically for quasi-periodic motion. This kind of procedure has been introduced in [23].

We could look for a 2-dimensional invariant torus in the form of a parametrization of the form

$$
\begin{aligned}
& \psi: \mathbb{R}^{2} \longrightarrow \mathbb{R}^{6} \\
& \left(\theta_{1}, \theta_{2}\right) \mapsto \psi\left(\theta_{1}, \theta_{2}\right),
\end{aligned}
$$


$\psi$ being a $2 \pi$-periodic function in the $\theta_{1}, \theta_{2}$ variables. We could find such a $\psi$ by solving the functional equation

$$
\psi\left(\theta_{1}+t \omega_{1}, \theta_{2}+t \omega_{2}\right)=\phi_{t}\left(\psi\left(\theta_{1}, \theta_{2}\right)\right), \quad \forall t \in \mathbb{R}, \quad \forall \theta_{1}, \theta_{2} \in[0,2 \pi],
$$

where $\omega_{1}, \omega_{2}$ are the frequencies of the torus, $\dagger$ and $\phi_{t}$ is the time- $t$ flow of Hill's problem in Hamiltonian form, that is

$$
\frac{\mathrm{d}}{\mathrm{d} t} \phi_{t}(x)=X_{\mathcal{H}}\left(\phi_{t}(x)\right), \quad \phi_{0}(x)=x .
$$

where $X_{\mathcal{H}}$ denotes the vectorfield associated to the Hamiltonian $\mathcal{H}$.

In order to reduce the dimension of the problem, as well as to eliminate the indeterminacy introduced by the $t$ variable in (16), we will not look for a parametrization of the whole torus but of an invariant curve on it. For instance, we can choose $\eta_{0} \in[0,2 \pi]$, and define $\varphi: \mathbb{R} \rightarrow \mathbb{R}^{6}$ by

$$
\varphi(\xi)=\psi\left(\xi, \eta_{0}\right)
$$

so that it parametrizes the $\left\{\theta_{2}=\eta_{0}\right\}$ invariant curve. It can be seen that $\varphi$ satisfies the following functional equation

$$
\varphi(\xi+\rho)=\phi_{\delta}(\varphi(\xi)), \quad \forall \xi \in[0,2 \pi],
$$

where $\delta=T_{2}=2 \pi / \omega_{2}$ is the period associated to the $\omega_{2}$ frequency, and $\rho=2 \pi \omega_{1} / \omega_{2}$ is the so-called rotation number of the invariant curve $\{\varphi(\xi)\}_{\xi \in[0,2 \pi]}$ with respect to the map $\phi_{\delta}$. It is also easy to see that the parametrization of the whole torus can be recovered from the parametrization of the invariant curve using

$$
\psi\left(\theta_{1}, \theta_{2}\right)=\phi_{\left(\left(\theta_{2}-\eta_{0}\right) / 2 \pi\right) \delta}\left(\varphi\left(\theta_{1}-\frac{\theta_{2}-\eta_{0}}{2 \pi} \rho\right)\right) .
$$

Indeed, from (18) it can be seen that $\psi$, defined as in (19), satisfies

$$
\phi_{t}\left(\psi\left(\theta_{1}, \theta_{2}\right)\right)=\psi\left(\left(\theta_{1}, \theta_{2}\right)+t\left(\frac{\rho}{\delta}, \frac{2 \pi}{\delta}\right)\right) .
$$

Therefore, we will look for $\varphi$ solving (18). In order to turn it into a finite system of equations, we will discretize both the parameter space and the function space. For the function space, we will look for $\varphi$ as a truncated Fourier series,

$$
\varphi(\xi)=A_{0}+\sum_{k=1}^{N_{f}}\left(A_{k} \cos (k \xi)+B_{k} \sin (k \xi)\right),
$$

where $A_{i}, B_{i} \in \mathbb{R}^{6}$. The criterion for determining $N_{f}$ will be discussed below. For the parameter space, we will discretize $[0,2 \pi]$ into $2 N_{f}+1$ equally spaced values,

$$
\xi_{i}=i \frac{2 \pi}{1+2 N_{f}}, \quad i=0, \ldots, 2 N_{f},
$$

and solve

$$
\varphi\left(\xi_{i}+\rho\right)=\phi_{\delta}\left(\varphi\left(\xi_{i}\right)\right), \quad i=0, \ldots, 2 N_{f}
$$

for $A_{0}, A_{1}, B_{1}, \ldots, A_{N_{f}}, B_{N_{f}}$.

$\dagger$ If we are close to the $L_{1}$ libration point, $\omega_{1}$ and $\omega_{2}$ will be close to the horizontal and vertical frequencies, $\omega$ and $\nu$, associated to the linear behaviour around $L_{1}$ given in (7). 
System (21) still has two drawbacks. Although, under generic conditions, for suitable and fixed values of $\delta$ and $\rho$ there exists a unique torus with the corresponding frequency vector $\left(\omega_{1}, \omega_{2}\right)$, system $(21)$ does not have unique solutions but rather a two-dimensional manifold of solutions. This is due to the fact that:

- if $\varphi(\xi)$ is a solution of (18), then, for any $\xi_{0} \in \mathbb{R}, \varphi\left(\xi-\xi_{0}\right)$ is a different function with a different Fourier series which is also a solution of (18), and

- through (17) we have chosen one invariant curve inside the torus, but there are as many of them as values of the $\eta_{0}$ parameter.

We can overcome the first source of indeterminacy, for instance, by fixing one coordinate of $A_{1}$ equal to zero (this makes sense as long as the corresponding coordinate of $B_{1}$ is different from zero). The second indetermination can be eliminated by fixing a coordinate of $A_{0}$, which has to be chosen by geometrical considerations.

The second drawback of (21) is that, due to the instability introduced by the saddle component of $L_{1}$, several significant digits are lost through numerical integration of a given initial condition during $\delta$ time units. To avoid this loss of precision, we use a multiple shooting strategy: instead of looking for a single $\varphi$ satisfying (21), we look for $\varphi_{0}=\varphi, \varphi_{1}, \ldots, \varphi_{m-1}$ satisfying

$$
\begin{aligned}
\varphi_{j+1}(\xi) & =\phi_{\delta / m}\left(\varphi_{j}(\xi)\right), \quad j=0 \div m-2 \\
\varphi_{0}(\xi+\rho) & =\phi_{\delta / m}\left(\varphi_{m-1}(\xi)\right) .
\end{aligned}
$$

Finally, due to the fact that the energy is a relevant parameter in our computations, we will introduce it in the set of unknowns, together with an additional equation in the system to be solved. In this way, we will solve

$$
\begin{aligned}
\mathcal{H}\left(\varphi_{0}(0)\right)-h & =0, \\
\varphi_{j+1}\left(\xi_{i}\right)-\phi_{\delta / m}\left(\varphi_{j}\left(\xi_{i}\right)\right) & =0, \quad j=0, \ldots, m-2, \quad i=0, \ldots, 2 N_{f}, \\
\varphi_{0}\left(\xi_{i}+\rho\right)-\phi_{\delta / m}\left(\varphi_{m-1}\left(\xi_{i}\right)\right) & =0, \quad i=0, \ldots, 2 N_{f},
\end{aligned}
$$

where the unknowns are

$$
h, \delta, \rho, A_{0}^{0}, A_{1}^{0}, B_{1}^{0}, \ldots, A_{N_{f}}^{0}, B_{N_{f}}^{0}, \ldots, A_{0}^{m-1}, A_{1}^{m-1}, B_{1}^{m-1}, \ldots, A_{N_{f}}^{m-1}, B_{N_{f}}^{m-1},
$$

with $h, \delta, \rho \in \mathbb{R}, A_{i}^{j}, B_{i}^{j} \in \mathbb{R}^{6}$ and

$$
\varphi_{j}(\xi)=A_{0}^{j}+\sum_{l=0}^{N_{f}}\left(A_{l}^{j} \cos (l \xi)+B_{l}^{j} \sin (l \xi)\right), \quad j=0, \ldots, m-1 .
$$

System (22) is solved both for the refinement of a single invariant torus and for the continuation of a one-parameter family of invariant tori. In both cases we keep constant one coordinate of $A_{0}^{0}$ and one coordinate of $A_{1}^{0}$ (or $B_{1}^{0}$ ), in order to eliminate the two indeterminations that we have already pointed out. Moreover:

- In order to refine a single invariant torus, we keep constant two unknowns among $h, \delta, \rho$. This is because the tori that we are looking for are embedded in 2-parameter families (see, for instance, figure 1, where, for a fixed value of the energy $(h=0.2)$, there is a one-parameter family of tori, starting at the vertical Lyapunov orbit and ending at the planar one).

- In order to continue a one-parametric family of invariant tori, we keep just one of the unknowns $h, \delta, \rho$ constant. 
For the refinement of a torus, we have used Newton's method, and for the continuation of a 1-parameter family, the standard predictor-corrector procedure used for the continuation of families of periodic orbits. Note that, after all the possibilities mentioned, we can end up with a linear system of equations that:

- can be square or not,

- can have unique solution or a kernel of known dimension.

To deal with all the different situations in a uniform way, what we compute is the minimum-norm least-squares solution of a not-necessarily square linear system, and optionally its kernel. For this goal we have used LAPACK's routine for $Q R$ decomposition with column pivoting [23, 24].

It must be noted that, unlike the case of periodic orbits, the families of invariant tori are not continuous but cantorian. Nevertheless, from the computational point of view we can treat them as if they were continuous, as long as the 'gaps' in the families are small enough, and this has been the situation that we have found.

A comment must be made on the choice of $N_{f}$. Following Castellà and Jorba [22], we estimate the discretization error as

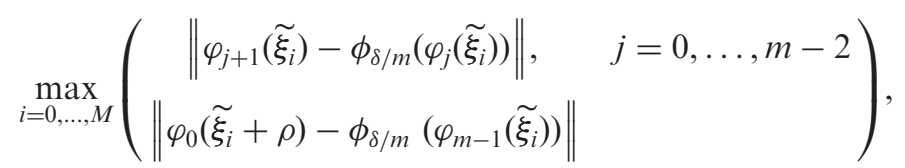

where $\tilde{\xi}_{i}=i 2 \pi / M$, for $M \gg 2 N_{f}+1$. So we take $N_{f}$ as large as needed in order to keep the previous estimate under a given tolerance. Note that this is the relevant error estimate if we plan to use (18) and (19) to integrate a trajectory on the torus for an arbitrary large time interval.

The only point that remains is to show how to get initial conditions of an invariant torus, to start the refinement and continuation procedures from a periodic orbit with central part. Let $x_{0}$ be an initial condition of such an orbit with period $T$. Assume that its monodromy matrix $M:=D \phi_{T}\left(x_{0}\right)$ has an eigenvalue of the form $e^{i v}$ with $v \in[0,2 \pi]$. Let $v_{1}+i v_{2}$ be an associated eigenvector. Denote the linear approximation of the time- $t$ flow around $x_{0}$ by

$$
L_{\phi_{t}}^{x_{0}}(x)=\phi_{t}\left(x_{0}\right)+M\left(x-x_{0}\right)
$$

and define

$$
\begin{aligned}
L_{\varphi}(\xi) & :=x_{0}+\gamma\left(v_{1} \cos \xi_{0}+v_{2} \sin \xi_{0}\right) \cos \xi+\gamma\left(v_{1} \sin \xi_{0}-v_{2} \cos \xi_{0}\right) \sin \xi \\
& =x_{0}+\left(v_{1}, v_{2}\right) R_{\xi-\xi_{0}}\left(\begin{array}{l}
\gamma \\
0
\end{array}\right),
\end{aligned}
$$

where $\left(v_{1}, v_{2}\right)$ is the $6 \times 2$ matrix with columns $v_{1}$ and $v_{2}$, and

$$
R_{\xi}=\left(\begin{array}{cc}
\cos \xi & \sin \xi \\
-\sin \xi & \cos \xi
\end{array}\right)
$$

Then, it is easy to check that

$$
L_{\varphi}(\xi+\nu)-L_{\phi_{T}}^{x_{0}}\left(L_{\varphi}(\xi)\right)=0,
$$


that is, $L_{\varphi}$ satisfies the linearization of equation (18) with $\rho=v, \delta=T$. Therefore, we can take as initial seed for the procedure

$$
\begin{aligned}
h & =\mathcal{H}\left(x_{0}\right), & A_{0} & =x_{0}, \\
\delta & =T, & A_{1} & =\gamma\left(v_{1} \cos \xi_{0}+v_{2} \sin \xi_{0}\right), \\
\rho & =v, & B_{1} & =\gamma\left(v_{1} \sin \xi_{0}-v_{2} \cos \xi_{0}\right), \\
& & A_{j}, B_{j} & =0 \quad(j \geq 2),
\end{aligned}
$$

where $\xi_{0}$ can be chosen in order to make one coordinate of $A_{1}$ be equal to zero, so that one of the indeterminations pointed out above is eliminated. Note that we have taken $\delta$ equal to the period of the p.o. There is also the possibility of taking an initial seed such that $\delta$ is equal to a normal period associated to the $e^{i v}$ eigenvalue, that is

$$
\delta=\frac{2 \pi}{v} T \text {. }
$$

More concretely, in analogy with equation (19), we can reconstruct from $L_{\varphi}$ a parametrization of a whole 2-dimensional torus invariant by the linear flow as

$$
L_{\psi}\left(\theta_{1}, \theta_{2}\right)=L_{\phi_{\left(\theta_{2} / 2 \pi\right) T}}^{x_{0}}\left(L_{\varphi}\left(\theta_{1}-\frac{\theta_{2}}{2 \pi} \nu\right)\right) .
$$

It can be checked that, with $L_{\psi}$ defined as above,

$$
L_{\phi_{t}}^{\phi_{\left(\theta_{2} / 2 \pi\right) T}\left(x_{0}\right)}\left(L_{\psi}\left(\theta_{1}, \theta_{2}\right)\right)=L_{\psi}\left(\left(\theta_{1}, \theta_{2}\right)+t\left(\frac{v}{T}, \frac{2 \pi}{T}\right)\right)
$$

The choice (25) of $\delta$ corresponds to the frequency of the $\theta_{1}$ variable in (26). Then, according to the deduction of (18) from (16), the corresponding $\rho$ must be

$$
\rho=\delta \frac{2 \pi}{T}=\frac{(2 \pi)^{2}}{v}
$$

Due to the fact that $v$ is determined up to an integer multiple of $2 \pi$, and also that both $e^{i v}$ and $e^{-i v}$ are eigenvalues of the monodromy matrix, $v$ can be substituted in all the above equations by $\pm v+2 \pi j$, for $j \in \mathbb{Z}$, so that we can take as initial seed

$$
\delta=\frac{2 \pi}{ \pm v+2 \pi j} T, \quad \rho=\frac{(2 \pi)^{2}}{ \pm v+2 \pi j} .
$$

The reader is refereed to $[19,26]$ for additional details concerning technical aspects of the implementation, including strategies to perform the computations in parallel on a Beowulf-class cluster.

\subsection{Continuation of families of invariant tori}

In this section, we make some comments on several families of invariant tori that have been computed with the above methodology.

6.2.1 Invariant tori around Lyapunov orbits. The full two-parameter family of invariant tori generated by the central part of the planar and vertical Lyapunov families of periodic orbits is represented schematically in figure 23. These invariant tori are the well-known Lissajous orbits. They are represented, in the $(h, \rho)$ plane, by the points inside the region delimited by the $\alpha, \beta$ and $\gamma$ curves. These points have coordinates 


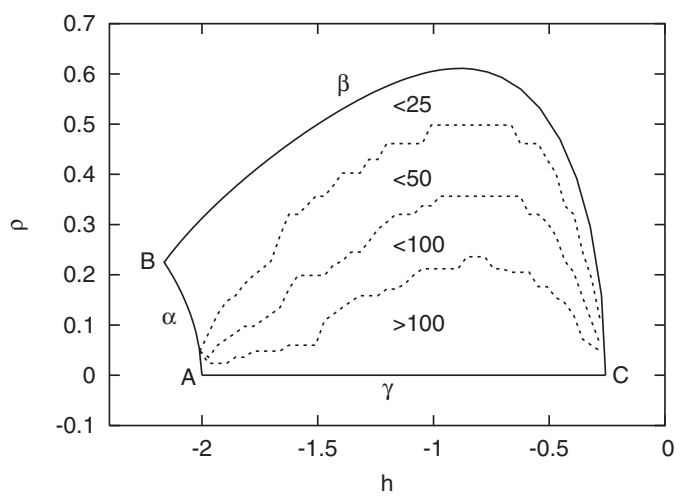

Figure 23. Region of the energy-rotation number plane covered by the twoparametric family of tori computed starting at the vertical and planar Lyapunov families of periodic orbits. Vertex $\mathrm{A}$ is at the value of the energy at which the halo families are born. Vertex B is at the value of the energy of the libration point. Vertex $\mathrm{C}$ is at the value of the energy of the first bifurcation of the vertical Lyapunov family.

$(h, \rho), h$ and $\rho$ being two of the unknowns (23) of the numerical procedure described in the previous section.

The $\beta$ curve represents the vertical Lyapunov family of periodic orbits. Its points are $\left(h, v_{V}\right)$, where $h$ is the energy level of each orbit (value of the Hamiltonian $\mathcal{H}$ ) and $v_{V}$ is the argument in $[0, \pi]$ of the eigenvalue of the monodromy matrix corresponding to its central part. The B point corresponds to $L_{1}$ point, where the Lyapunov periodic families are born. The $\mathrm{C}$ point corresponds to the first bifurcation of the family, in which it loses its central part. In the following, we will denote as $\left(h_{2}, \rho_{2}\right)$ the coordinates of the B point of figure 23 .

The upper half of the region of invariant tori $\left\{\rho>\rho_{2}\right\}$ has been computed by starting from several vertical p.o. according to (24), with $v=v_{V}$ and $T=T_{V}$, where $T_{V}$ is the period of the vertical orbit. The corresponding initial approximations of invariant tori have been refined and then continued by keeping $\rho$ fixed. Each of these uniparametric families of invariant tori (with constant $\rho$ ) would be seen in figure 23 as a horizontal line, which would end at the intersection with the $\beta$ curve to the right of the figure. This intersection represents the end of the family at a vertical Lyapunov p.o. of a higher energy level than the starting one but the same $v_{V}$.

We can also continue iso-energetic families of invariant tori (keeping $h$ fixed instead of $\rho$ ). In figure 24 we represent some tori of one of these continuations. We start at a vertical Lyapunov orbit according to (24). As we continue with $h$ fixed, $\rho$ decreases until we end at a planar Lyapunov p.o. At this termination, the $\delta$ and $\rho$ parameters are numerically checked to be

$$
\delta=\frac{2 \pi}{2 \pi-v_{P}} T_{P}, \quad \rho=\frac{(2 \pi)^{2}}{2 \pi-v_{P}}-2 \pi,
$$

where $T_{P}$ is the period of the ending planar orbit and $v_{P}$ is the argument in $[0, \pi]$ of the eigenvalue of the monodromy matrix of the p.o. corresponding to its central part. These values correspond to those given in (27) taking the minus sign for $v$ and $j=1$. 

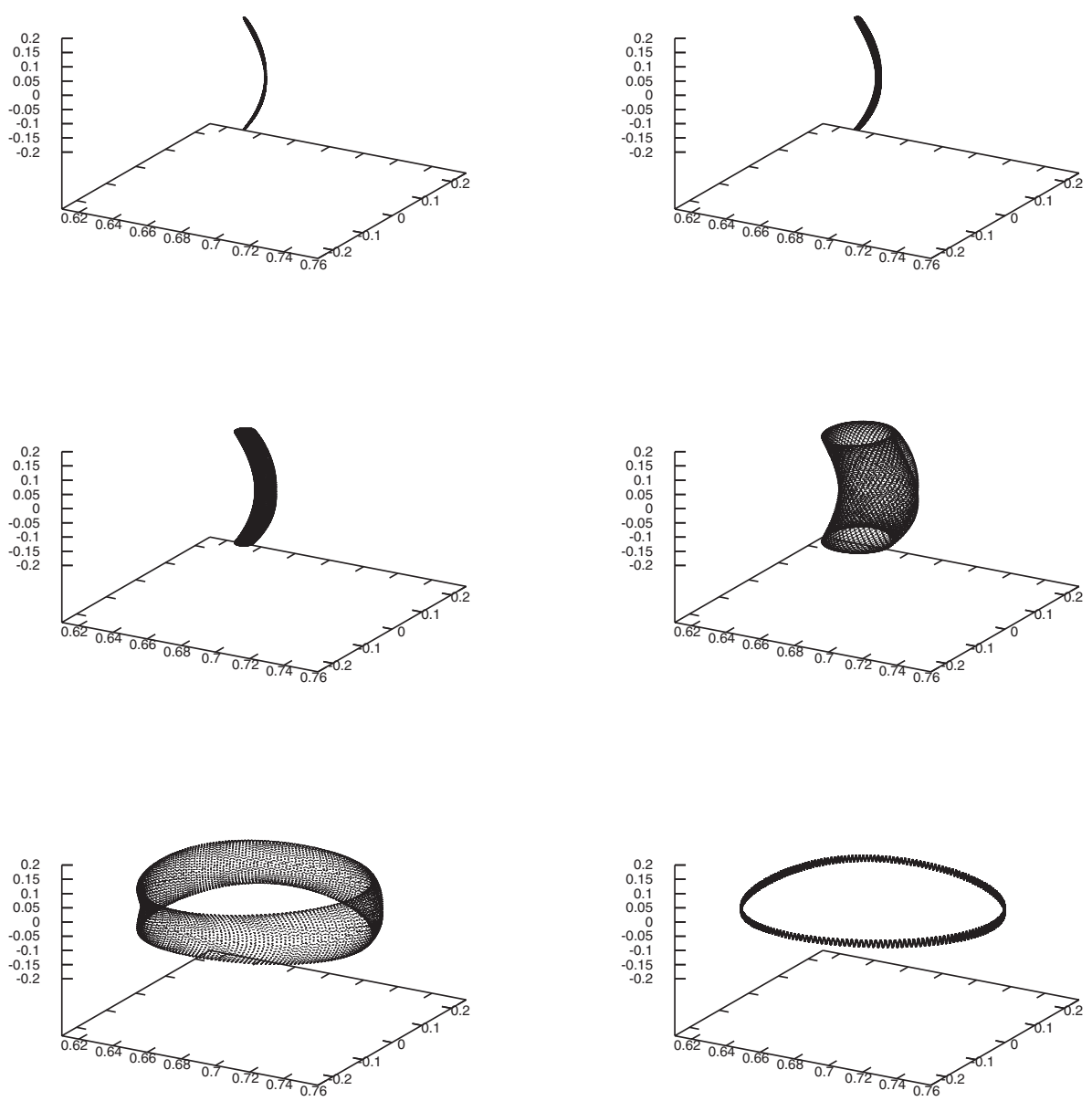

Figure 24. Isoenergetic family of 2D tori starting at a vertical Lyapunov orbit and ending at a planar one. Energy level: -2.08645 .

Therefore, we can represent the planar Lyapunov family in figure 23, together with the vertical one, as $\left(h,\left((2 \pi)^{2} /\left(2 \pi-v_{P}\right)\right)-2 \pi\right)$. These points correspond to the $\alpha$ curve.

In order to compute the lower half $\left(\rho<\rho_{2}\right)$ of the region of invariant tori, it is more convenient to do the continuations by keeping $\rho$ constant instead of $h$, since in this case we can avoid the crossing of low-order resonances (there are no such resonances in the continuation of figure 24, but they are found for higher energy levels). According to this, we have started from the planar Lyapunov orbits using (27), taking $v=v_{P}$ with the minus sign and $j=1$. As with the upper half of the region, these continuations would also be seen as horizontal lines that end at the intersection with a vertical Lyapunov orbit of a higher energy level but the same $\rho$.

The bottom curve $\gamma$ of figure 23 represents the separatrix between the Lyapunov family of invariant tori and the invariant tori around halo orbits, which starts at the energy level in which halo orbits appear (the A point). One of the points of this curve has been computed in section 2.2 for $h=0.2$. 
Let us now relate the values of $(h, \rho)$ in the previous figure to the natural horizontal and vertical frequencies around the $L_{1}$ point. We can characterize each torus of the family by two frequencies, which will be denoted as vertical $\left(\omega_{V}\right)$ and planar $\left(\omega_{P}\right)$. For a given torus, its frequency vector $\omega=\left(\omega_{V}, \omega_{P}\right)$ is determined up to unimodular transformations $U$, in the sense that $U \omega$ can be used in a Fourier expansion of any quasi-periodic trajectory on it. For every admissible frequency vector, a natural choice is the one for which, when we continuously move along the family up to the equilibrium point $L_{1}$, the frequency vector $\omega=\left(\omega_{V}, \omega_{P}\right)$ tends to the frequency vector corresponding to the centre $\times$ centre part of the linearization of the flow around $L_{1}$, that is, $\bar{\omega}:=$ $\left(\bar{\omega}_{V}, \bar{\omega}_{P}\right)=(2, \sqrt{2 \sqrt{7}-1}=2.07159 \ldots)$. This is what we mean by the 'natural' horizontal and vertical frequencies, and these are precisely the ones physically observed as motions in configuration space.

Let $T_{P}, T_{V}, v_{P}$ and $v_{V}$ be defined as above, and denote by $\bar{T}_{P}, \bar{T}_{V}, \bar{v}_{P}$ and $\bar{v}_{V}$ the corresponding limiting values when we move along the Lyapunov families of p.o. towards the equilibrium point $L_{1}$. Using the linear behaviour of the flow around $L_{1}$ we obtain

$$
\begin{aligned}
& \bar{v}_{V}=2 \pi\left(\frac{\bar{\omega}_{P}}{\bar{\omega}_{V}}-1\right), \\
& \bar{v}_{P}=2 \pi\left(1-\frac{\bar{\omega}_{V}}{\bar{\omega}_{P}}\right) .
\end{aligned}
$$

From these two equations, we get

$$
\bar{v}_{V}=\frac{(2 \pi)^{2}}{2 \pi-\bar{v}_{P}}-2 \pi .
$$

Also, isolating $2 \pi / \bar{\omega}_{V}$ from (30) (which equals $\bar{T}_{V}$ ), we get

$$
\bar{T}_{V}=\frac{2 \pi}{2 \pi-\bar{v}_{P}} \bar{T}_{P}
$$

Note that (31) and (32) correspond to the choice (28) of $\delta$ and $\rho$ when we start from the planar Lyapunov family. From this fact, equation (29) and the continuity argument that defines $\left(\omega_{V}, \omega_{P}\right)$, we have that for every torus in figure 23 ,

$$
\delta=\frac{2 \pi}{\omega_{V}}, \quad \rho=2 \pi\left(\frac{\omega_{P}}{\omega_{V}}-1\right) .
$$

More concretely, from (20) and (26), the frequencies $\omega_{V}, \omega_{H}$ defined by (33) can be considered as frequencies of the torus being computed. By the above argument, they tend to $\bar{\omega}_{V}$ and $\bar{\omega}_{H}$ when we continuously move towards $L_{1}$ along the Lyapunov family of tori.

6.2.2 Invariant tori around halo and halo-type orbits. We have also computed the families of invariant tori that originate, in a suitable energy range, from the following families of periodic orbits:

- The halo family of p.o., from its bifurcation from the planar Lyapunov family to the turning point of the small stability parameter (see figure 6).

- The hyperbolic-elliptic bifurcation of the halo family by period tripling, in the first energy range for which the small stability parameter goes from 2 to -2 . 

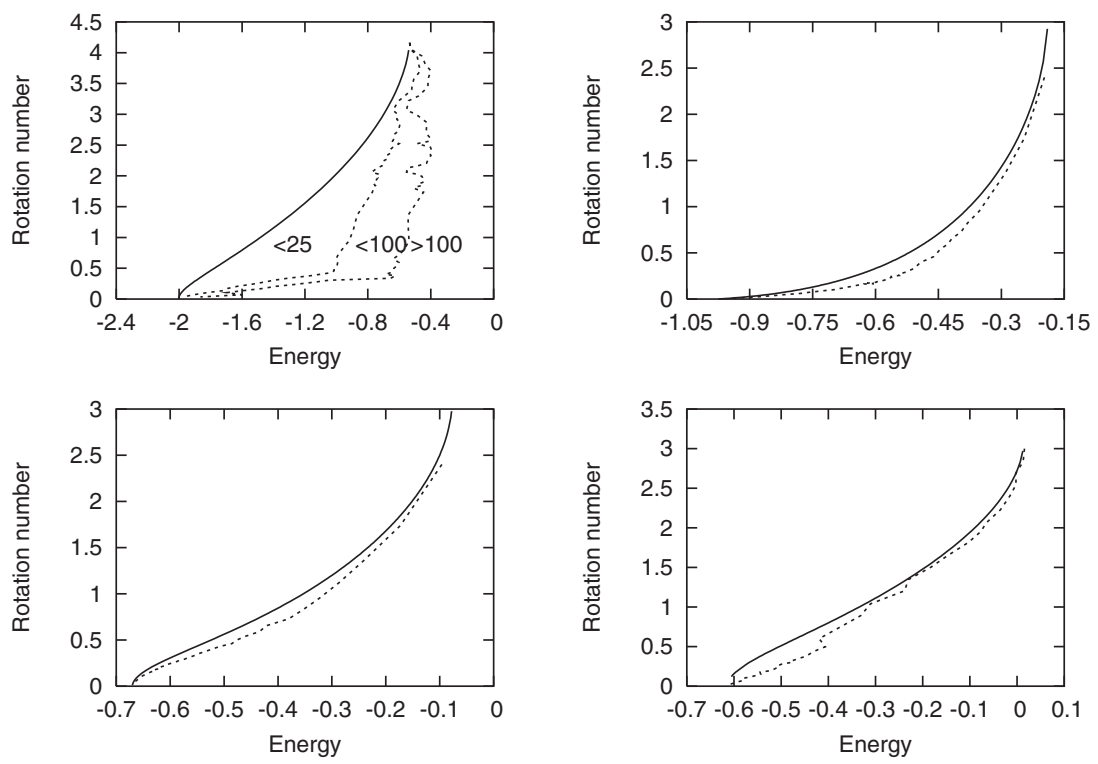

Figure 25. Regions in the energy-rotation number plane covered by the twoparameter families of invariant tori around halo orbits (top left), the elliptic families bifurcated from halo orbits by period tripling (top right), by period doubling (bottom left) and around planar orbits after the bifurcation of the bridge (bottom right).

- The hyperbolic-elliptic bifurcation of the halo family by period doubling, in the first energy range for which the small stability parameter goes from 2 to -2 .

- The Lyapunov planar family, from the energy level of the bifurcation of the two lane bridge to the energy in which the small stability parameter crosses -2 (see figure 4 ).

The corresponding $(h, \rho)$ diagrams for all these families of invariant tori are given in figure 25. In all cases the families of invariant tori have been continued with fixed $\rho$, starting from the backbone family of p.o. according to (24). Unlike the case of the previous section, these diagrams are not closed, and the continuation has been stopped when a given maximum number of harmonics (usually 100) has been reached.

The right-hand side of the region for the tori around halo orbits, which corresponds to reaching the maximum number of harmonics, detects the crossing of the rotation number $\rho$ through low-order resonances, at which the tori collapse to periodic orbits. The three main 'gulfs' detected correspond to the values of the rotation number equal to $2 \pi / 2,4 \pi / 5,2 \pi / 3$ (from top to bottom). The first and third peaks correspond to the $1: 2$ and $1: 3$ resonances related to the bifurcating families of halo-type orbits already mentioned.

\subsection{Poincaré sections of the centre manifold}

In order to compare the results obtained here with the ones computed using the reduction to the centre manifold, we will show in this section the evolution with 

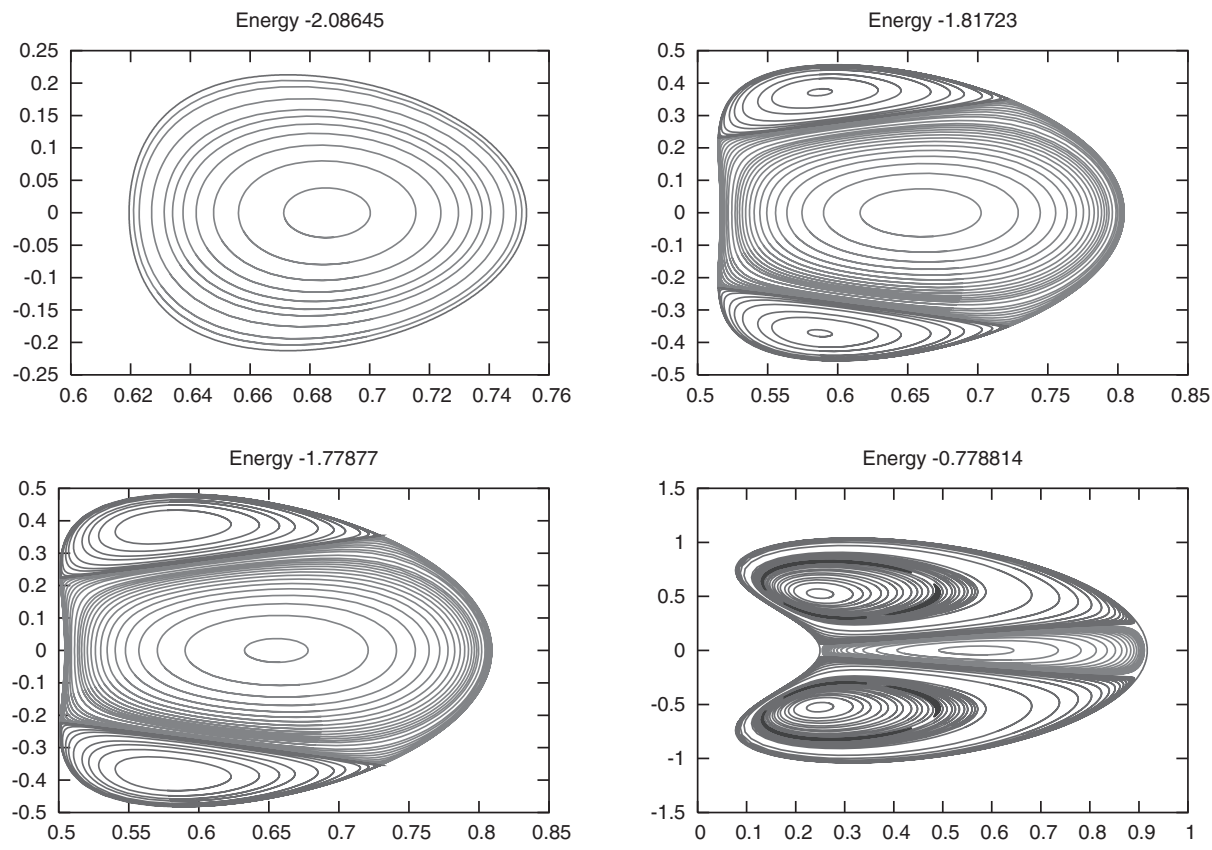

Energy -0.586514

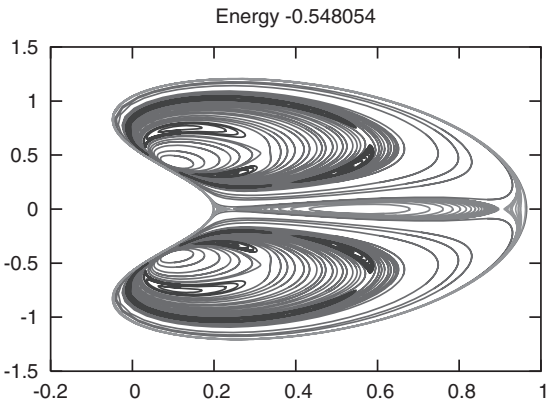

Figure 26. Energy slices of the section $z=0, p_{z}>0$ of the invariant tori around $L_{1}$ computed in the previous section.

respect to the energy of the behaviour of the Poincare sections through $z=0, p_{z}>0$, of all the different sorts of orbits computed. These Poincare sections are displayed in figure 26.

All the plots in figure 26 have a similar structure. The exterior curve is the Lyapunov planar orbit of the corresponding energy level. As this orbit is planar, it is completely included in the surface of section, and is the only orbit for which this happens. The motion inside the region bounded by the Lyapunov planar orbit is quasi-periodic, except at some gaps which cannot be distinguished in the picture. In all plots there is a fixed point on the $x$-axis, associated to the vertical Lyapunov orbit.

For small energy values, the entire picture is formed by invariant curves surrounding the fixed point associated to the vertical orbit. They are associated to the intersections of the Lissajous type trajectories around the vertical periodic orbit, whose evolution from the planar Lyapunov orbit to the vertical one was displayed in figure 24. At the energy level associated to the first bifurcation of the 

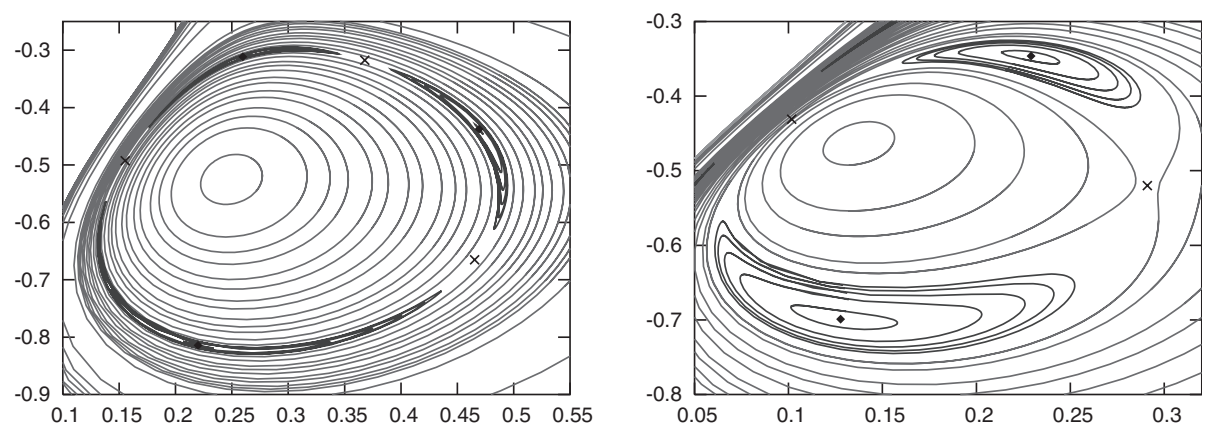

Figure 27. Magnification of the Poincaré sections of figure 26 of energy values -0.778814 (left) and -0.586514 (right). The $\diamond$ points represent the hyperbolic-elliptic families of p.o. bifurcated from the halo family by period tripling (left) and period doubling (right). The $\times$ points represent the hyperbolic-hyperbolic ones.

Lyapunov planar family, the halo orbits appear. This can be seen clearly in the Poincaré map representations, since there appear two additional fixed points surrounded by invariant curves. Increasing the values of the energy, the family of halo orbits has two relevant bifurcations, by period tripling and period doubling. Both bifurcations can be detected in the Poincare representations. As has already been mentioned, within the bifurcated families there are some with central part, which are surrounded by invariant tori. These tori give rise to the 'island chain' structure typical of two-dimensional area-preserving maps. To display this behaviour more clearly, we show in figure 27 a magnification of the bifurcated periodic orbits and its surrounding invariant tori.

The region between the tori around the vertical Lyapunov orbit and the tori around the halo orbits is not empty, as it appears to be the case in the above figures, and should contain the traces, on the Poincare map representation, of the invariant manifold of the Lyapunov planar orbit. These manifolds act as separatrices between the two types of motion. The same thing happens between the islands of the bifurcated halo-type orbits and the tori around the halo orbits. In this case, the region between the two types of tori is filled with the traces of the invariant manifolds of the bifurcated hyperbolic halo-type orbits. In all these boundary regions, the motion should have a chaotic behaviour.

For the last two energy values, the two lane bridge joining the planar and vertical Lyapunov families has already bifurcated, so the planar family has gained a central part and, therefore, its periodic orbits are surrounded by invariant tori. The $\{z=0\}$ sections of these tori are the most outer curves that appear in the last two plots of figure 26. The invariant manifolds of these bifurcated p.o. are the ones that must act as separatrices between the different kinds of tori for these values of the energy.

In figures 28 and 29, we represent a sample torus of each of the families computed.

\section{Conclusions}

In this paper, we have shown how semi-analytical and numerical techniques can be combined to get non-local information of the flow of the 3-dimensional 

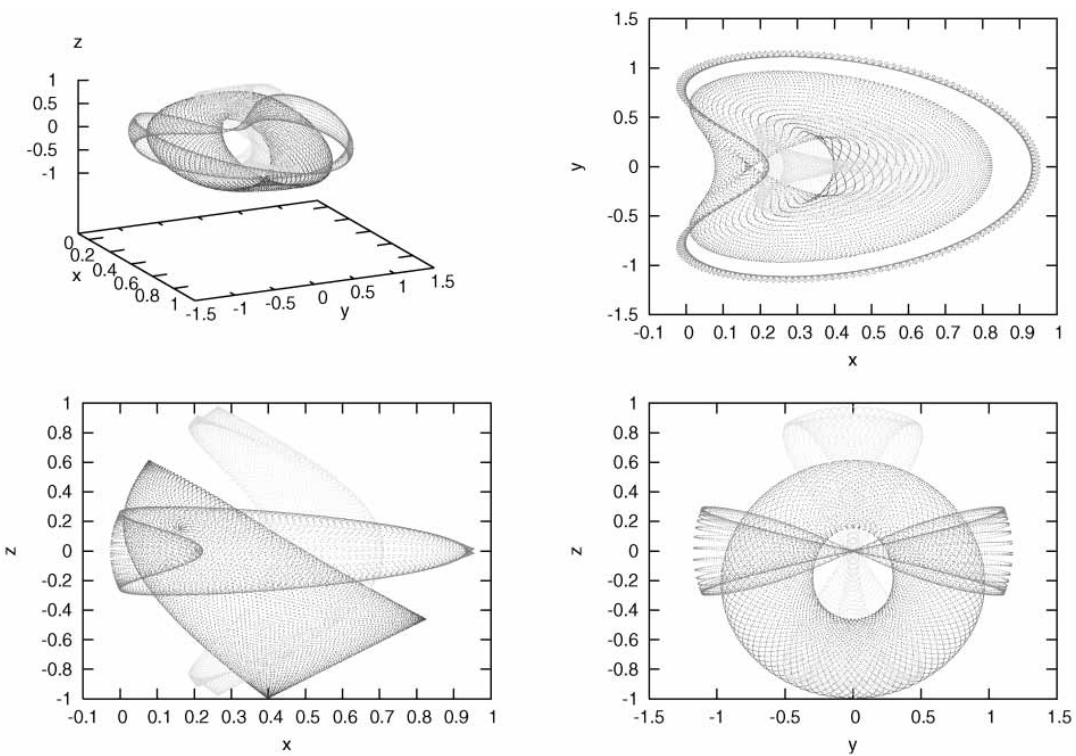

Figure 28. Sample torus, for the energy value -0.586514 , around each of the following families of p.o.: planar Lyapunov family after the bifurcation of the bridge, halo family and vertical Lyapunov family.
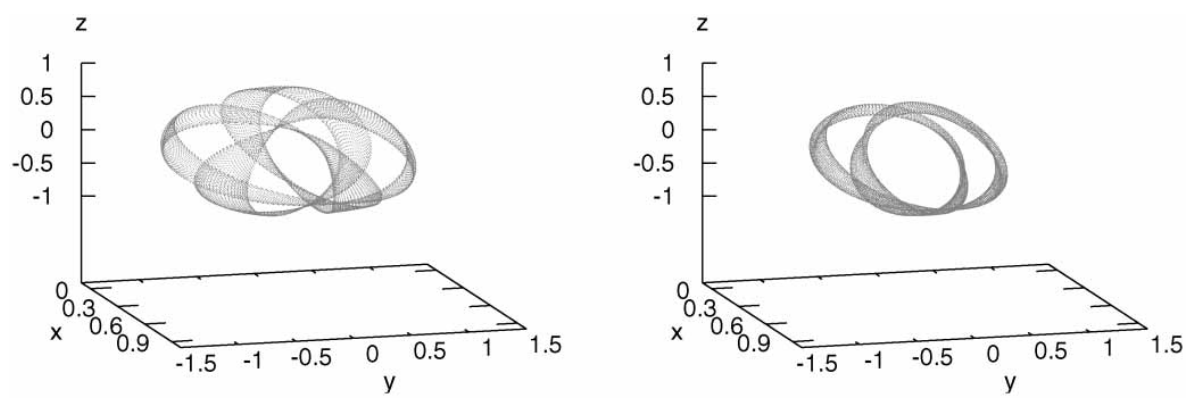

Figure 29. For the energy value -0.586514 , a sample torus around the hyperbolicelliptic family of p.o. bifurcated from the halo one by period tripling (left) and period doubling (right).

Hill's problem. They are applied to get a complete description of the libration orbits around an extended neighbourhood of the collinear libration points, as well as their invariant manifolds. The two approaches have their own limitations, convergence problems and large CPU time computations, but with the use of both a relatively large interval of energy values can be explored.

\section{Acknowledgements}

This work has been partially supported by grant CIRIT 2001 SGR-70 (Catalonia) and grant BFM2003-09504-C02-01 (MCYT, Spain). M.M. acknowledges the support of the doctoral research grant AP2001-3064 (MECD, Spain). 


\section{References}

[1] Conley, C.C., 1968, Low energy transit orbits in the restricted three-body problem. SIAM Journal on Applied Mathematics, 16(4), 732-746.

[2] Gómez, G. and Masdemont, J.J., 2000, Some zero cost transfers between halo orbits. Advances in the Astronautical Sciences, 115, 1199-1216.

[3] Koon, W.S., Lo, M.W., Marsden, J.E. and Ross, S.D., 2000, Heteroclinic connections between periodic orbits and resonance transitions in celestial mechanics. Chaos, 10, 427-496.

[4] Gómez, G., Koon, W.S., Lo, M.W., Marsden, J.E., Masdemont, J.J. and Ross, S.D., 2004, Connecting orbits and invariant manifolds in the spatial restricted three-body problem. Nonlinearity, 17, 1571-1606.

[5] Gómez, G., Jorba, À., Masdemont, J.J. and Simó, C., 1993, Study of the transfer from the earth to a halo orbit around the equilibrium point $L_{1}$. Celestial Mechanics and Dynamical Astronomy, 56, $541-562$.

[6] Gómez, G., Jorba, À., Masdemont, J.J. and Simó, C., 1998, Study of the transfer between halo aorbits. Acta Astronautica, 43, 493-520.

[7] Koon, W.S., Lo, M.W., Marsden, J.E. and Ross, S.D., 1999, The genesis trajectory and heteroclinic connections. AAS Paper 99-451, AAS/AIAA Astrodynamics Specialist Conference, Girdwood, Alaska

[8] Hénon, M., 1969, Numerical exploration of the restricted problem. V. Hill's case: periodic orbits and their stability. Astronomy \& Astrophysics, 1, 223-238.

[9] Simó, C. and Stuchi, T., 2000, Central stable/unstable manifolds and the destruction of KAM tori in the planar Hill problem. Physica D, 140, 1-32.

[10] Szebehely, V., 1967, Theory of Orbits (New York: Academic Press).

[11] Hill, G.W., 1878, Researches in the lunar theory. American Journal of Mathematics, 1, 5-26, 129-147, 245-260.

[12] Wintner, A., 1941, Analytical Foundations of Celestial Mechanics (Princeton, NJ: Princeton University Press).

[13] Stiefel, E.L. and Scheifele, G., 1971, Linear and Regular Celestial Mechanics (Berlin: Springer-Verlag).

[14] Meletlidou, E., Ichtiaroglou, S. and Winterberg, J., 2001, Non-integrability of Hill's lunar problem. Celestial Mechanics and Dynamical Astronomy, 80, 145-156.

[15] Winterberg, J. and Meletlidou, E., 2004, Non-continuation of integrals of the rotating two-body problem in Hill's lunar problem. Celestial Mechanics and Dynamical Astronomy, 88, 37-49.

[16] Jorba, A. and Masdemont, J.J., 1999, Dynamics in the center manifold of the restricted three-body problem. Physica D, 132, 189-213.

[17] Gómez, G., Jorba, À., Masdemont, J.J. and Simó, C., 2000, Dynamics and Mission Design Near Libration Point Orbits-Volume 3: Advanced Methods for Collinear Points (Singapore: World Scientific).

[18] Dichmann, D.J., Doedel, E.J. and Paffenroth, R.C., 2003, The computation of periodic solutions of the 3-body problem using the numerical continuation software AUTO. In: G. Gómez, M.W. Lo and J.J. Masdemont (Eds.), Libration Point Orbits and Applications, pp. 489-528 (Singapore: World Scientific).

[19] Gómez, G. and Mondelo, J.M., 2001, The dynamics around the collinear equilibrium points of the RTBP. Physica D, 157, 283-321.

[20] Siegel, C.L. and Moser, J.K., 1971, Lectures on Celestial Mechanics (Berlin: Springer-Verlag).

[21] Hénon, M., 1973, Vertical stability of periodic orbits in the restricted problem. Astronomy and Astrophysics, 28, 415-426.

[22] Marcote, M., 2005, PhD Universitat de Barcelona (in preparation).

[23] Castellà, E. and Jorba, Á., 2000, On the vertical families of two-dimensional tori near the triangular points of the bicircular problem. Celestial Mechanics and Dynamical Astronomy, 76, 35-54.

[24] Anderson, E., Bai, Z., Bischof, C., Demmel, J., Dongarra, J., Croz, D., Greenbaum, A., Hammarling, S., McKenney, A., Ostrouchov, S. and Sorensen, D., 1995, LAPACK Users' Guide, 22nd Edn., (SIAM).

[25] Golub, G.H. and Van Loani, C.R., 1996, Matrix Computations, 3rd Edn (Baltimore, MD: The Johns Hopkins University Press).

[26] Mondelo, J.M., 2001, Contribution to the study of Fourier methods for quasi-periodic functions and the vicinity of the collinear librations points. PhD thesis, Universitat de Barcelona.

[27] Hénon, M., 2003, New families of periodic orbits in Hill's problem of three bodies. Celestial Mechanics and Dynamical Astronomy, 85, 223-246. 